\title{
Demazure Character Formulas for Generalized Kac-Moody Algebras
}

\author{
Motohiro Ishii \\ Graduate School of Pure and Applied Sciences, University of Tsukuba, \\ Tsukuba, Ibaraki 305-8571, Japan \\ (e-mail: ishii731@math.tsukuba.ac.jp)
}

\begin{abstract}
For a dominant integral weight $\lambda$, we introduce a family of $U_{q}^{+}(\mathfrak{g})$-submodules $V_{w}(\lambda)$ of the irreducible highest weight $U_{q}(\mathfrak{g})$-module $V(\lambda)$ of highest weight $\lambda$ for a generalized Kac-Moody algebra $\mathfrak{g}$. We prove that the module $V_{w}(\lambda)$ is spanned by its global basis, and then give a character formula for $V_{w}(\lambda)$, which generalizes the Demazure character formula for ordinary Kac-Moody algebras.
\end{abstract}

\section{Introduction}

For a (symmetrizable) Kac-Moody algebra $\mathfrak{g}$, the Demazure character formula describes the (formal) character of a Demazure module, which is a $U^{+}(\mathfrak{g})$-submodule generated by an extremal weight vector of an integrable highest weight $U(\mathfrak{g})$-module; this formula was proved by Kumar ([Kum]) and Mathieu ([M]) independently by using geometric methods. Also, from an algebraic viewpoint, it is possible to formulate the notion of Demazure modules for an integrable highest weight $U_{q}(\mathfrak{g})$-module. In fact, Littelmann $([\mathbf{L i 3}])$ gave a (conjectural) algebraic description of the Demazure character formula in terms of Kashiwara's crystal bases for $\mathfrak{g}$ of finite type. Soon afterward, this conjecture of Littelmann was proved by Kashiwara ([Kas2]) generally for a KacMoody algebra. More precisely, Kashiwara showed that a Demazure module is spanned by its global basis, and that there exists a basis at the crystal limit " $q=0$ ", which is called a Demazure crystal.

In $[\mathbf{J K K}, \mathbf{J K K S}]$, Kashiwara's crystal basis theory was extended to the case of generalized Kac-Moody algebras. Also, Littelmann's path model for representations of Kac-Moody algebras was extended to the case of generalized Kac-Moody algebras by Joseph and Lamprou ([JL]). Therefore, it is natural to expect the existence of Demazure crystals for an irreducible highest weight module over a generalized Kac-Moody algebra. The purpose of this paper is to obtain a generalization of Demazure modules, their crystal bases, and a character formula for them for generalized Kac-Moody algebras.

It is known that the representation theory of generalized Kac-Moody algebras are very similar to that of ordinary Kac-Moody algebras, and many results for Kac-Moody algebras are extended 
to the case of generalized Kac-Moody algebras. However, there are some obstructions, which come from the existence of imaginary simple roots, for the study of the structure of an irreducible highest weight module over a generalized Kac-Moody algebra. Recall that a Demazure crystal decomposes into a disjoint union of $\mathfrak{s l}_{2}$-strings of finite length in the case of ordinary KacMoody algebras. In contrast, in the case of generalized Kac-Moody algebras, $\mathfrak{s l}_{2}$-strings and "Heisenberg algebra"-strings corresponding to imaginary simple roots for an irreducible highest weight module are no longer of finite length. Hence, we cannot apply a method similar to the case of ordinary Kac-Moody algebras to our setting. To overcome this difficulty, we introduce a certain Kac-Moody algebra $\tilde{\mathfrak{g}}$ defined from a given Borcherds-Cartan datum of a generalized Kac-Moody algebra $\mathfrak{g}$, and then we relate the representation theory of $\mathfrak{g}$ to the one of $\tilde{\mathfrak{g}}$. This enables us to study the structure of an irreducible highest weight module over a generalized Kac-Moody algebra by comparing it with the corresponding one over an ordinary Kac-Moody algebra. In this way, under a certain condition, we can define Demazure modules for a generalized Kac-Moody algebra, and show that they are spanned by their global bases (Theorem 3). As a result, we obtain a character formula for these Demazure modules for a generalized Kac-Moody algebra by introducing a "modified" Demazure operator for each imaginary simple root (Theorem 4).

Let us state our results more precisely. Let $\mathfrak{g}$ be a generalized Kac-Moody algebra and $\tilde{\mathfrak{g}}$ the associated Kac-Moody algebra (see $\S 2.2$ ). For a dominant integral weight $\lambda \in P^{+}$for $\mathfrak{g}$ and the corresponding dominant integral weight $\tilde{\lambda} \in \widetilde{P}^{+}$for $\tilde{\mathfrak{g}}$ (see $\S 2.5$ ), we denote by $\mathbb{B}(\lambda)$ and $\widetilde{\mathbb{B}}(\tilde{\lambda})$ the sets of generalized Lakshmibai-Seshadri paths and ordinary Lakshmibai-Seshadri paths, respectively. Then, there exists an embedding $\mathbb{B}(\lambda) \hookrightarrow \widetilde{\mathbb{B}}(\tilde{\lambda})$ of path crystals (Proposition 2.5.1); note that this is not a morphism of crystals. By using this embedding, we have the following decomposition rules for $U_{q}(\mathfrak{g})$-modules in the category $\mathcal{O}_{\text {int }}$ (see $[\mathbf{J K K}, \S 2]$ ). Denote by $V(\lambda)$ the irreducible highest weight $U_{q}(\mathfrak{g})$-module of highest weight $\lambda \in P^{+}$.

Theorem 1 Let $\lambda, \mu \in P^{+}$be dominant integral weights for $\mathfrak{g}$. Then, we have an isomorphism of $U_{q}(\mathfrak{g})$-modules:

$$
V(\lambda) \otimes V(\mu) \cong \bigoplus_{\substack{\pi \in \mathbb{B}(\mu) \\ \tilde{\pi}: \tilde{\lambda} \text {-dominant }}} V(\lambda+\pi(1)) .
$$

Here, $\tilde{\pi} \in \widetilde{\mathbb{B}}(\tilde{\mu})$ denotes the image of $\pi \in \mathbb{B}(\mu)$ under the embedding $\mathbb{B}(\mu) \hookrightarrow \widetilde{\mathbb{B}}(\tilde{\mu})$, and it is said to be $\tilde{\lambda}$-dominant if $\tilde{\pi}(t)+\tilde{\lambda}$ belongs to the dominant Weyl chamber of $\tilde{\mathfrak{g}}$ for all $t \in[0,1]$.

Theorem 2 Let $\lambda \in P^{+}$. For a subset $S$ of the index set $I$ of simple roots of $\mathfrak{g}$, we denote by $\mathfrak{g}_{S}$ the corresponding Levi subalgebra of $\mathfrak{g}$, by $\tilde{\mathfrak{g}}_{\widetilde{S}}$ the corresponding one for $\tilde{\mathfrak{g}}$ (see $§ 3.1$ ), and by 
$V_{S}(\mu)$ the irreducible highest weight $U_{q}\left(\mathfrak{g}_{S}\right)$-module of highest weight $\mu$. Then,

$$
V(\lambda) \cong \bigoplus_{\substack{\pi \in \mathbb{B}(\lambda) \\ \tilde{\pi}: \tilde{\mathfrak{g}}_{\widetilde{S}} \text {-dominant }}} V_{S}(\pi(1)) \text { as } U_{q}\left(\mathfrak{g}_{S}\right) \text {-modules. }
$$

Here, $\tilde{\pi} \in \widetilde{\mathbb{B}}(\tilde{\lambda})$ denotes the image of $\pi \in \mathbb{B}(\lambda)$ under the embedding $\mathbb{B}(\lambda) \hookrightarrow \widetilde{\mathbb{B}}(\tilde{\lambda})$, and it is said to be $\tilde{\mathfrak{g}}_{\widetilde{S}^{-}}$dominant if $\tilde{\pi}(t)$ belongs to the dominant Weyl chamber of $\tilde{\mathfrak{g}}_{\widetilde{S}}$ for all $t \in[0,1]$.

Also, we prove an analog of the Parthasarathy-Ranga Rao-Varadarajan conjecture for generalized Kac-Moody algebras (Theorem 3.2.4), using the embedding of path models and the tensor product decomposition rule (Theorem 1).

Now, let us describe our main results. For a dominant integral weight $\lambda \in P^{+}$, we take and fix an element $w$ in the monoid $\mathcal{W}$ (see Definition 2.3.1) satisfying the condition:

there exists an expression $w=v_{l} r_{j_{l}} \cdots v_{1} r_{j_{1}} v_{0}$, with $j_{1}, \ldots, j_{l} \in I^{i m}$ and $v_{0}, v_{1}, \ldots, v_{l} \in$ $\mathcal{W}_{\text {re }}($ see $\S 2.1$ and $\S 2.3)$, such that

$$
\alpha_{j_{s}}^{\vee}\left(v_{s-1} r_{j_{s-1}} \cdots v_{1} r_{j_{1}} v_{0}(\lambda)\right)=1 \text { for all } s=1,2, \ldots, l,
$$

where $\alpha_{j}^{\vee}, j \in I$, are the simple coroots of $\mathfrak{g}$.

For such an element $w \in \mathcal{W}$, we set $V_{w}(\lambda):=U_{q}^{+}(\mathfrak{g}) V(\lambda)_{w \lambda}$, where $V(\lambda)_{\mu} \subset V(\lambda)$ is the weight space of weight $\mu \in P$.

Theorem 3 For $\lambda \in P^{+}$and $w \in \mathcal{W}$ satisfying the condition above, there exists a subset $B_{w}(\lambda) \subset B(\lambda)$ of the crystal basis $B(\lambda)$ of $V(\lambda)$ such that

$$
V_{w}(\lambda)=\bigoplus_{b \in B_{w}(\lambda)} \mathbb{C}(q) G_{\lambda}(b)
$$

where $\left\{G_{\lambda}(b)\right\}_{b \in B(\lambda)}$ denotes the global basis of $V(\lambda)$.

For the element $w \in \mathcal{W}$ above, we can take a specific expression, a minimal dominant reduced expression (see $\S 4.2$ ), $w=w_{k} r_{i_{k}}^{a_{k}} \cdots w_{1} r_{i_{1}}^{a_{1}} w_{0}$, with $w_{0}, w_{1}, \ldots, w_{k} \in \mathcal{W}_{r e}, a_{1}, \ldots, a_{k} \in \mathbb{Z}_{>0}$, and $i_{1}, \ldots, i_{k} \in I^{i m}$ (all distinct). For each $i \in I^{r e}$, we define an operator $\mathcal{D}_{i}$ on the group ring $\mathbb{Z}[P]:=\bigoplus_{\mu \in P} \mathbb{Z} e^{\mu}$ of the weight lattice $P$ of $\mathfrak{g}$ by:

$$
\mathcal{D}_{i}\left(e^{\mu}\right):=\frac{e^{\mu}-e^{\mu-\left(1+\alpha_{i}^{\vee}(\mu)\right) \alpha_{i}}}{1-e^{-\alpha_{i}}} .
$$

For a reduced expression $v=r_{j_{l}} \cdots r_{j_{2}} r_{j_{1}} \in \mathcal{W}_{r e}$, with $j_{1}, j_{2}, \ldots, j_{l} \in I^{r e}$, we set $\mathcal{D}_{v}:=$ $\mathcal{D}_{j_{l}} \cdots \mathcal{D}_{j_{2}} \mathcal{D}_{j_{1}}$. Also, for each $i \in I^{i m}$ and $a \in \mathbb{Z}_{\geq 1}$, we define an operator $\mathcal{D}_{i}^{(a)}$ by

$$
\mathcal{D}_{i}^{(a)}\left(e^{\mu}\right):= \begin{cases}e^{\mu} & \text { if } \alpha_{i}^{\vee}(\mu)=0, \\ \sum_{m=0}^{a} e^{\mu-m \alpha_{i}} & \text { otherwise. }\end{cases}
$$


Theorem 4 Let $\lambda \in P^{+}$and $w=w_{k} r_{i_{k}}^{a_{k}} \cdots w_{1} r_{i_{1}}^{a_{1}} w_{0} \in \mathcal{W}$ be as above. Then, the (formal) character of the Demazure module $V_{w}(\lambda)$ is given as follows:

$$
\operatorname{ch} V_{w}(\lambda)=\mathcal{D}_{w_{k}} \mathcal{D}_{i_{k}}^{\left(a_{k}\right)} \cdots \mathcal{D}_{w_{1}} \mathcal{D}_{i_{1}}^{\left(a_{1}\right)} \mathcal{D}_{w_{0}}\left(e^{\lambda}\right)
$$

This paper is organized as follows. In Section 2, we recall some elementary facts about generalized Kac-Moody algebras and Joseph-Lamprou's path model. Also, we review the construction of an embedding of Joseph-Lamprou's path model into Littelmann's path model. In Section 3, we give proofs of Theorems 1 and 2, and show an analog of the Parthasarathy-RangaRao-Varadarajan conjecture for generalized Kac-Moody algebras. In Section 4, we introduce Demazure modules for generalized Kac-Moody algebras, and study the structure of them. Finally in Section 5, we prove Theorems 3 and 4 .

\section{Acknowledgments}

The author is grateful to Professor Satoshi Naito for reading the manuscript very carefully and for valuable comments. He is also grateful to Professor Daisuke Sagaki for valuable comments. The author's research was supported by the Japan Society for the Promotion of Science Research Fellowships for Young Scientists.

\section{Preliminaries}

\subsection{Generalized Kac-Moody algebras}

In this subsection, we recall some fundamental facts about generalized Kac-Moody algebras. For more details, we refer the reader to [Bo, JKK, JKKS, JL, Kac, Kan].

Let $I$ be a (finite or) countable index set. We call $A=\left(a_{i j}\right)_{i, j \in I}$ a Borcherds-Cartan matrix if the following three conditions are satisfied: (1) $a_{i i}=2$ or $a_{i i} \in \mathbb{Z}_{\leq 0}$ for each $i \in I ;(2) a_{i j} \in \mathbb{Z}_{\leq 0}$ for all $i, j \in I$ with $i \neq j$; (3) $a_{i j}=0$ if and only if $a_{j i}=0$ for all $i, j \in I$ with $i \neq j$.

An index $i \in I$ is said to be real if $a_{i i}=2$, and imaginary if $a_{i i} \leq 0$. Denote by $I^{r e}:=\{i \in$ $\left.I \mid a_{i i}=2\right\}$ the set of real indices, and by $I^{i m}:=\left\{i \in I \mid a_{i i} \leq 0\right\}=I \backslash I^{r e}$ the set of imaginary indices. A Borcherds-Cartan matrix $A$ is said to be symmetrizable if there exists a diagonal matrix $D=\operatorname{diag}\left(d_{i}\right)_{i \in I}$, with $d_{i} \in \mathbb{Z}_{>0}$, such that $D A$ is symmetric. Also, if $a_{i i} \in 2 \mathbb{Z}$ for all $i \in I$, then $A$ is said to be even. Throughout this paper, we assume that the Borcherds-Cartan matrix is symmetrizable and even.

For a given Borcherds-Cartan matrix $A=\left(a_{i j}\right)_{i, j \in I}$, a Borcherds-Cartan datum is a quintuple $\left(A, \Pi:=\left\{\alpha_{i}\right\}_{i \in I}, \Pi^{\vee}:=\left\{\alpha_{i}^{\vee}\right\}_{i \in I}, P, P^{\vee}\right)$, where $\Pi$ and $\Pi^{\vee}$ are the sets of simple roots and simple coroots, respectively, $P^{\vee}$ is a coweight lattice, and $P:=\operatorname{Hom}_{\mathbb{Z}}\left(P^{\vee}, \mathbb{Z}\right)$ is a weight lattice. We set 
$\mathfrak{h}:=P^{\vee} \otimes_{\mathbb{Z}} \mathbb{C}$, and call it the Cartan subalgebra. Let $\mathfrak{h}^{*}:=\operatorname{Hom}_{\mathbb{C}}(\mathfrak{h}, \mathbb{C})$ denote the full dual space of $\mathfrak{h}$. In this paper, we assume that $\Pi^{\vee} \subset \mathfrak{h}$ and $\Pi \subset \mathfrak{h}^{*}$ are both linearly independent over $\mathbb{C}$. Let $P^{+}:=\left\{\lambda \in P \mid \alpha_{i}^{\vee}(\lambda) \geq 0\right.$ for all $\left.i \in I\right\}$ be the set of dominant integral weights, and $Q:=\bigoplus_{i \in I} \mathbb{Z} \alpha_{i}$ the root lattice; we set $Q^{+}:=\sum_{i \in I} \mathbb{Z}_{\geq 0} \alpha_{i}$. Let $\mathfrak{g}$ be the generalized KacMoody algebra associated with a Borcherds-Cartan datum $\left(A, \Pi, \Pi^{\vee}, P, P^{\vee}\right)$. We have the root space decomposition $\mathfrak{g}=\bigoplus_{\alpha \in \mathfrak{h}^{*}} \mathfrak{g}_{\alpha}$, where $\mathfrak{g}_{\alpha}:=\{x \in \mathfrak{g} \mid \operatorname{ad}(h)(x)=h(\alpha) x$ for all $h \in \mathfrak{h}\}$, and $\mathfrak{h}=\mathfrak{g}_{0}$. Denote by $\Delta:=\left\{\alpha \in \mathfrak{h}^{*} \mid \mathfrak{g}_{\alpha} \neq\{0\}, \alpha \neq 0\right\}$ the set of roots, and by $\Delta^{+}$the set of positive roots. Note that $\Delta \subset Q \subset P$ and $\Delta=\Delta^{+} \sqcup\left(-\Delta^{+}\right)$. Also, we define a Coxeter group $\mathcal{W}_{r e}:=\left\langle r_{i} \mid i \in I^{r e}\right\rangle_{\text {group }} \subset \mathrm{GL}\left(\mathfrak{h}^{*}\right)$, where $r_{i}$ for $i \in I^{r e}$ is a simple reflection, and set $\Delta_{i m}:=\mathcal{W}_{r e} \Pi_{i m}$, where $\Pi_{i m}:=\left\{\alpha_{i}\right\}_{i \in I^{i m}}$ is the set of imaginary simple roots; note that $\Delta_{i m} \subset \Delta^{+}$. In addition, we set $\Delta_{r e}:=\mathcal{W}_{r e} \Pi_{r e}$ and $\Delta_{r e}^{+}:=\Delta_{r e} \cap \Delta^{+}$, where $\Pi_{r e}:=\left\{\alpha_{i}\right\}_{i \in I^{r e}}$ is the set of real simple roots. As in the case of ordinary Kac-Moody algebras, it is easily checked that the coroot $\beta^{\vee}:=w \alpha_{i}^{\vee}$ of $\beta=w \alpha_{i} \in \Delta_{r e}^{+} \sqcup \Delta_{i m}$ is well-defined (see [JL, §2.1.9]).

Definition 2.1.1 Let $q$ be an indeterminate. The quantized universal enveloping algebra $U_{q}(\mathfrak{g})$ associated with a Borcherds-Cartan datum $\left(A, \Pi, \Pi^{\vee}, P, P^{\vee}\right)$, with $D=\operatorname{diag}\left(d_{i}\right)_{i \in I}$ as above, is $a \mathbb{C}(q)$-algebra generated by the symbols $e_{i}, f_{i}, i \in I$, and $q^{h}, h \in P^{\vee}$, subject to the following relations:

$$
\begin{aligned}
& \cdot q^{0}=1, q^{h_{1}} q^{h_{2}}=q^{h_{1}+h_{2}} \text { for } h_{1}, h_{2} \in P^{\vee}, \\
& \cdot \quad q^{h} e_{i} q^{-h}=q^{h\left(\alpha_{i}\right)} e_{i}, q^{h} f_{i} q^{-h}=q^{-h\left(\alpha_{i}\right)} f_{i} \text { for } h \in P^{\vee} \text { and } i \in I, \\
& \cdot \quad\left[e_{i}, f_{j}\right]=\delta_{i j} \frac{K_{i}-K_{i}^{-1}}{q_{i}-q_{i}^{-1}} \text { for } i, j \in I \text {, where we set } q_{i}:=q^{d_{i}} \text { and } K_{i}:=q^{d_{i} \alpha_{i}^{\vee},} \\
& \cdot \sum_{r=0}^{1-a_{i j}}(-1)^{r}\left[\begin{array}{c}
1-a_{i j} \\
r
\end{array}\right]_{i} e_{i}^{1-a_{i j}-r} e_{j} e_{i}^{r}=0 \text { for } i \in I^{r e} \text { and } j \in I, \text { with } i \neq j, \\
& \cdot \sum_{r=0}^{1-a_{i j}}(-1)^{r}\left[\begin{array}{c}
1-a_{i j} \\
r
\end{array}\right]_{i} f_{i}^{1-a_{i j}-r} f_{j} f_{i}^{r}=0 \text { for } i \in I^{r e} \text { and } j \in I \text {, with } i \neq j, \\
& \cdot \quad\left[e_{i}, e_{j}\right]=\left[f_{i}, f_{j}\right]=0 \text { if } a_{i j}=0 .
\end{aligned}
$$

Here, we set $[n]_{i}:=\frac{q_{i}^{n}-q_{i}^{-n}}{q_{i}-q_{i}^{-1}},[n]_{i} !:=\prod_{k=1}^{n}[k]_{i}$, and $\left[\begin{array}{c}m \\ n\end{array}\right]_{i}:=\frac{[m]_{i} !}{[m-n]_{i} ![n]_{i} !}$ for $i \in I$.

If $a_{i i}<0$, then we set $c_{i}:=-\frac{1}{2} a_{i i} \in \mathbb{Z}_{>0}$, and define $\{n\}_{i}:=\frac{q_{i}^{c_{i} n}-q_{i}^{-c_{i} n}}{q_{i}^{c_{i}}-q_{i}^{-c_{i}}}$. If $a_{i i}=0$, we set $\{n\}_{i}:=n$. We define divided powers by $e_{i}^{(n)}:=\frac{e_{i}^{n}}{[n]_{i} !}, f_{i}^{(n)}:=\frac{f_{i}^{n}}{[n]_{i} !}$ if $i \in I^{r e}$, and by $e_{i}^{(n)}:=e_{i}^{n}$, $f_{i}^{(n)}:=f_{i}^{n}$ if $i \in I^{i m}$. Here we understand that $e_{i}^{(0)}=f_{i}^{(0)}:=1$, and $e_{i}^{(n)}=f_{i}^{(n)}:=0$ for 
$n<0$. Let $U_{q}^{+}(\mathfrak{g})$ and $U_{q}^{-}(\mathfrak{g})$ be the subalgebras of $U_{q}(\mathfrak{g})$ generated by $e_{i}, i \in I$, and $f_{i}, i \in I$, respectively.

Let $V(\lambda)$ be the irreducible highest weight $U_{q}(\mathfrak{g})$-module of highest weight $\lambda \in P^{+}$. We define the Kashiwara operators $\tilde{e}_{i}, \tilde{f}_{i}, i \in I$, on $V(\lambda)$ in the following way (see [JKK, §4]). Since we have $V(\lambda)=\bigoplus_{n \geq 0} f_{i}^{(n)} \operatorname{Ker}\left(e_{i}\right)$ for each $i \in I$, and the weight space decomposition $V(\lambda)=$ $\bigoplus_{\mu \in P} V(\lambda)_{\mu}$, each weight vector $v \in V(\lambda)_{\mu}, \mu \in P$, has the unique expression $v=\sum_{n \geq 0} f_{i}^{(n)} v_{n}$ such that (a) $v_{n} \in \operatorname{Ker}\left(e_{i}\right) \cap V(\lambda)_{\mu+n \alpha_{i}}$, (b) if $i \in I^{r e}$ and $\alpha_{i}^{\vee}\left(\mu+n \alpha_{i}\right)<n$, then $v_{n}=0$, and (c) if $i \in I^{i m}, n>0$, and $\alpha_{i}^{\vee}\left(\mu+n \alpha_{i}\right)=0$, then $v_{n}=0$. This expression for $v$ is called the $i$-string decomposition. Then, we define $\tilde{f}_{i} v:=\sum_{n \geq 0} f_{i}^{(n+1)} v_{n}$ and $\tilde{e}_{i} v:=\sum_{n \geq 1} f_{i}^{(n-1)} v_{n}$. Note that if $i \in I^{i m}$, then $\tilde{f}_{i}=f_{i}$.

Let $u_{\lambda} \in V(\lambda)_{\lambda} \backslash\{0\}$ denote the highest weight vector, and $B(\lambda)$ the crystal basis of $V(\lambda)$ with the crystal lattice $L(\lambda)$. Here, $L(\lambda)$ is a free module over the local ring $\{f(q) / g(q) \mid f(q), g(q) \in$ $\mathbb{C}[q], g(0) \neq 0\}$ generated by $\tilde{f}_{i_{l}} \cdots \tilde{f}_{i_{1}} u_{\lambda}, l \geq 0, i_{1}, \ldots, i_{l} \in I$, and we have $B(\lambda)=\left\{\tilde{f}_{i_{l}} \cdots \tilde{f}_{i_{1}} u_{\lambda}\right.$ $\left.\bmod q L(\lambda) \mid l \geq 0, i_{1}, \ldots, i_{l} \in I\right\} \backslash\{0\}$. Let $\mathbf{A}:=\mathbb{C}\left[q, q^{-1}\right]$, and denote by $V(\lambda)^{\mathbf{A}}$ the $\mathbf{A}$-form of $V(\lambda)$. Let ${ }^{-}: U_{q}(\mathfrak{g}) \rightarrow U_{q}(\mathfrak{g})$ be the $\mathbb{C}$-algebra automorphism defined by

$$
q \longmapsto q^{-1}, q^{h} \longmapsto q^{-h}, e_{i} \longmapsto e_{i}, f_{i} \longmapsto f_{i},
$$

for $h \in P^{\vee}$ and $i \in I$. Also, we define a $\mathbb{C}$-linear automorphism ${ }^{-}$on $V(\lambda)$ by $\overline{X u_{\lambda}}:=\bar{X} u_{\lambda}$ for $X \in U_{q}(\mathfrak{g})$. Let $\left\{G_{\lambda}(b)\right\}_{b \in B(\lambda)}$ denote the global basis of $V(\lambda)$. We know from $[\mathbf{J K K}$, Theorem 9.3] that the element $G_{\lambda}(b), b \in B(\lambda)$, is characterized by the following three conditions: (i) $\overline{G_{\lambda}(b)}=G_{\lambda}(b)$, (ii) $G_{\lambda}(b) \in V(\lambda)^{\mathbf{A}} \cap L(\lambda)$, and (iii) $G_{\lambda}(b) \equiv b \bmod q L(\lambda)$.

\subsection{The associated Kac-Moody algebra $\tilde{\mathfrak{g}}$}

We associate a Cartan matrix $\widetilde{A}$ with a given Borcherds-Cartan matrix $A=\left(a_{i j}\right)_{i, j \in I}$ as follows. Set $\tilde{I}:=\{(i, 1)\}_{i \in I^{r e}} \sqcup\{(i, m)\}_{i \in I^{i m}, m \in \mathbb{Z}_{\geq 1}}$, and define a Cartan matrix $\widetilde{A}:=\left(\tilde{a}_{(i, m),(j, n)}\right)_{(i, m),(j, n) \in \tilde{I}}$ by

$$
\left\{\begin{array}{l}
\tilde{a}_{(i, m),(i, m)}:=2 \text { for }(i, m) \in \tilde{I} \\
\tilde{a}_{(i, m),(j, n)}:=a_{i j} \text { for }(i, m),(j, n) \in \tilde{I} \text { with }(i, m) \neq(j, n) .
\end{array}\right.
$$

Note that if $A$ is symmetrizable, then so is $\widetilde{A}$ (see $[\mathbf{I}$, Lemma 4.1.1]). Let us denote by $(\widetilde{A}, \widetilde{\Pi}:=$ $\left.\left\{\tilde{\alpha}_{(i, m)}\right\}_{(i, m) \in \tilde{I}}, \widetilde{\Pi}^{\vee}:=\left\{\tilde{\alpha}_{(i, m)}^{\vee}\right\}_{(i, m) \in \tilde{I}}, \widetilde{P}, \widetilde{P}^{\vee}\right)$ the Cartan datum associated with $\widetilde{A}$, where $\widetilde{\Pi}, \widetilde{\Pi}^{\vee}$ are the sets of simple roots and simple coroots, respectively, $\widetilde{P}$ is a weight lattice, and $\widetilde{P}^{\vee}$ is a coweight lattice. Let $\tilde{\mathfrak{g}}$ be the associated Kac-Moody algebra, $\tilde{\mathfrak{h}}:=\widetilde{P}^{\vee} \otimes_{\mathbb{Z}} \mathbb{C}$ the Cartan subalgebra, and $\widetilde{W}$ the Weyl group. Note that every permutation on the subset $\{(i, m)\}_{m \in \mathbb{Z}_{\geq 1}} \subset$ $\tilde{I}$ induces a (Dynkin) diagram automorphism of $\tilde{\mathfrak{g}}$ for each $i \in I^{i m}$. We denote by $\mathfrak{S}_{i}$ the permutation group on the subset $\{(i, m)\}_{m \in \mathbb{Z}_{\geq 1}} \subset \tilde{I}$ for each $i \in I^{i m}$, and set $\Omega:=\prod_{i \in I^{i m}} \mathfrak{S}_{i}$. 
For notational simplicity, we write $(\mathbf{i}, \mathbf{m}):=\left(\left(i_{s}, m_{s}\right)\right)_{s=1}^{k}=\left(\left(i_{k}, m_{k}\right), \ldots,\left(i_{2}, m_{2}\right),\left(i_{1}, m_{1}\right)\right)$ if $\mathbf{i}=\left(i_{k}, \ldots, i_{2}, i_{1}\right) \in I^{k}$ and $\mathbf{m}=\left(m_{k}, \ldots, m_{2}, m_{1}\right) \in \mathbb{Z}^{k}$ for $k \geq 0$, and call it an ordered index if $m_{r}=1$ for $i_{r} \in I^{r e}$, and if $m_{x_{s}}=s$ for all $s=1,2, \ldots, t$, where $\left\{x_{1}, x_{2}, \ldots, x_{t}\right\}=\{1 \leq x \leq k \mid$ $\left.i_{x}=i\right\}$, with $1 \leq x_{1}<x_{2}<\cdots<x_{t} \leq k$, for each $i \in I^{i m}$. We set $\mathcal{I}:=\bigcup_{k=1}^{\infty} I^{k}, \widetilde{\mathcal{I}}:=\bigcup_{k=1}^{\infty} \tilde{I}^{k}$, and denote by $\widetilde{\mathcal{I}}_{\text {ord }}$ the set of all ordered indices. Since the $\mathbf{m}$ for which $(\mathbf{i}, \mathbf{m}) \in \widetilde{\mathcal{I}}_{\text {ord }}$ is determined uniquely by $\mathbf{i}$, we have a bijection $\mathcal{I} \rightarrow \widetilde{\mathcal{I}}_{\text {ord }}, \mathbf{i} \mapsto(\mathbf{i}, \mathbf{m})$. Note that $\Omega$ acts on $\tilde{\mathcal{I}}$ diagonally.

Example 2.2.1 If $I=\{1, \mathbf{2}, \mathbf{3}\}, I^{r e}=\{1\}$ and $I^{i m}=\{\mathbf{2}, \mathbf{3}\}$, then $\tilde{I}=\{(1,1)\} \sqcup\{(\mathbf{2}, m)$, $(\mathbf{3}, m)\}_{m \in \mathbb{Z}_{\geq 1}}$. An ordered index $(\mathbf{i}, \mathbf{m}) \in \widetilde{\mathcal{I}}_{\text {ord }}$ corresponding to $\mathbf{i}=(\mathbf{3}, 1, \mathbf{3}, \mathbf{3}, 1, \mathbf{2}, \mathbf{3}, 1, \mathbf{3}, \mathbf{2}, 1, \mathbf{2}) \in$ $\mathcal{I}$ is as follows:

$$
(\mathbf{i}, \mathbf{m})=((\mathbf{3}, 5),(1,1),(\mathbf{3}, 4),(\mathbf{3}, 3),(1,1),(\mathbf{2}, 3),(\mathbf{3}, 2),(1,1),(\mathbf{3}, 1),(\mathbf{2}, 2),(1,1),(\mathbf{2}, 1)) .
$$

We also define the subset $\widetilde{\mathcal{I}}_{\text {gen }} \subset \widetilde{\mathcal{I}}$ of generic indices in $\widetilde{\mathcal{I}}$ by $\widetilde{\mathcal{I}}_{\text {gen }}:=\left\{(\mathbf{i}, \mathbf{m})=\left(\left(i_{s}, m_{s}\right)\right)_{s=1}^{k} \in\right.$ $\widetilde{\mathcal{I}} \mid m_{s} \neq m_{t}$ for $s \neq t$ with $\left.i_{s}=i_{t} \in I^{i m}\right\}$; note that $\widetilde{\mathcal{I}}_{\text {ord }} \subset \widetilde{\mathcal{I}}_{\text {gen }} \subset \widetilde{\mathcal{I}}$, and that $\widetilde{\mathcal{I}}_{\text {gen }}=\Omega \widetilde{\mathcal{I}}_{\text {ord }}$. In particular, $\widetilde{\mathcal{I}}_{\text {gen }}$ is stable under the action of $\Omega$.

\subsection{The monoid $\mathcal{W}$}

In this subsection, we give a brief review of the monoid $\mathcal{W}$ and its properties. For more details, we refer the reader to $[\mathbf{I}, \S 2.2]$.

If we define $r_{i} \in \operatorname{GL}\left(\mathfrak{h}^{*}\right), i \in I^{i m}$, by $r_{i}(\mu):=\mu-\alpha_{i}^{\vee}(\mu) \alpha_{i}$ for $\mu \in \mathfrak{h}^{*}$, then the inverse of $r_{i}$ is given by

$$
r_{i}^{-1}(\mu)=\mu+\frac{1}{1-a_{i i}} \alpha_{i}^{\vee}(\mu) \alpha_{i} \text { for } \mu \in \mathfrak{h}^{*} .
$$

Note that this $r_{i}$ has an infinite order in $\operatorname{GL}\left(\mathfrak{h}^{*}\right)$.

Definition 2.3.1 ([I, Definition 2.2.1]). Let $\mathcal{W}$ denote the monoid generated by the symbols $\tilde{r}_{i}, i \in I$, subject to the following relations:

(1) $\tilde{r}_{i}^{2}=1$ for all $i \in I^{\text {re }}$;

(2) if $i, j \in I^{r e}, i \neq j$, and the order of $r_{i} r_{j} \in \mathrm{GL}\left(\mathfrak{h}^{*}\right)$ is $m \in\{2,3,4,6\}$, then we have

$$
\left(\tilde{r}_{i} \tilde{r}_{j}\right)^{m}=\left(\tilde{r}_{j} \tilde{r}_{i}\right)^{m}=1
$$

(3) if $i \in I^{i m}$, then for all $j \in I \backslash\{i\}$ such that $a_{i j}=0$, we have $\tilde{r}_{i} \tilde{r}_{j}=\tilde{r}_{j} \tilde{r}_{i}$.

Each element $w \in \mathcal{W}$ can be written as a product $w=\tilde{r}_{i_{1}} \tilde{r}_{i_{2}} \cdots \tilde{r}_{i_{k}}$ of generators $\tilde{r}_{i}, i \in I$. If the number $k$ is minimal among all the expressions for $w$ of the form above, then $k$ is called the length of $w$ and the expression $\tilde{r}_{i_{1}} \tilde{r}_{i_{2}} \cdots \tilde{r}_{i_{k}}$ is called a reduced expression. In this case, we write $\ell(w)=k$. Since the $r_{i} \in \mathrm{GL}\left(\mathfrak{h}^{*}\right), i \in I$, satisfy the conditions (1), (2) and (3) in Definition 2.3.1, we have the following (well-defined) homomorphism of monoids: 


$$
\mathcal{W} \longrightarrow \mathrm{GL}\left(\mathfrak{h}^{*}\right), \tilde{r}_{i} \longmapsto r_{i}, \text { for } i \in I \text {. }
$$

For simplicity, we write $r_{i}$ for $\tilde{r}_{i}$ in $\mathcal{W}$. Remark that $\mathcal{W}_{\text {re }}$ and $\left\langle r_{i} \mid i \in I^{r e}\right\rangle_{\text {monoid }} \subset \mathcal{W}$ are isomorphic as groups, where $\left\langle r_{i} \mid i \in I^{r e}\right\rangle_{\text {monoid }}$ denotes the submonoid of $\mathcal{W}$ generated by $r_{i}$, $i \in I^{r e}$; note that this submonoid is in fact a group by Definition 2.3.1 (1). Hence we may (and do) regard the group $\mathcal{W}_{\text {re }}$ as a submonoid of $\mathcal{W}$. For $\beta=w \alpha_{i} \in \Delta_{r e}^{+} \sqcup \Delta_{i m}, i \in I, w \in \mathcal{W}_{\text {re }}$, the element $r_{\beta}:=w r_{i} w^{-1}$ is well-defined (see $[\mathbf{I}, \S 2.2]$ ).

Definition 2.3.2 ([I, Definition 2.2.6]). For $w \in \mathcal{W}$ and $\beta \in \Delta_{r e}^{+} \sqcup \Delta_{\text {im }}$, we write $w \rightarrow r_{\beta} w$ if $\ell\left(r_{\beta} w\right)>\ell(w)$. Also, we define a partial order $\leq$ on $\mathcal{W}$ as follows: $w \leq w^{\prime}$ in $\mathcal{W}$ if there exist $w_{0}, w_{1}, \ldots, w_{l} \in \mathcal{W}$ such that $w=w_{0} \rightarrow w_{1} \rightarrow \cdots \rightarrow w_{l}=w^{\prime}$.

Note that if $v \leq w$ in $\mathcal{W}$ and $w=r_{i_{l}} \cdots r_{i_{2}} r_{i_{1}}$ is a reduced expression, then there exists a reduced expression $v=r_{i_{x_{p}}} \cdots r_{i_{x_{2}}} r_{i_{x_{1}}}, l \geq x_{p}>\cdots>x_{2}>x_{1} \geq 1$, by the Exchange Property of $\mathcal{W}$ (see $[\mathbf{I}, \S 2.2])$. However, the converse does not hold in general. For example, if $i, j \in I^{i m}$ with $a_{i j} \neq 0$, then $r_{i}$ and $r_{i} r_{j}$ are not comparable even though $r_{i}$ is a subword of $r_{i} r_{j}$ and each of them is a (unique) reduced expression.

Definition 2.3.3 ([I, Definition 2.2.10]). Let $w=w_{k} r_{i_{k}} \cdots w_{1} r_{i_{1}} w_{0} \in \mathcal{W}$, with $i_{1}, \ldots, i_{k} \in I^{i m}$ and $w_{0}, w_{1}, \ldots, w_{k} \in \mathcal{W}_{r e}$, be a reduced expression, namely, $\ell(w)=\ell\left(w_{k}\right)+1+\cdots+\ell\left(w_{1}\right)+1+$ $\ell\left(w_{0}\right)$. We call this expression a dominant reduced expression if it satisfies

$$
r_{i_{s}} w_{s-1} r_{i_{s-1}} \cdots w_{1} r_{i_{1}} w_{0}\left(P^{+}\right) \subset P^{+} \text {for all } s=1,2, \ldots, k \text {. }
$$

Note that every $w \in \mathcal{W}$ has at least one dominant reduced expression. Indeed, if we choose an expression of the form above in such a way that the sequence $\left(\ell\left(w_{0}\right), \ell\left(w_{1}\right), \ldots, \ell\left(w_{k}\right)\right)$ is minimal in lexicographic order among all the reduced expressions of the form above, then it is a dominant reduced expression (see $[\mathbf{I}, \S 2.2]$ ).

\subsection{Joseph-Lamprou's path model}

In this subsection, following [JL], we review Joseph-Lamprou's path model for generalized KacMoody algebras. For more details, we refer the reader to $[\mathbf{J L}, \mathbf{I}]$.

Let $\mathfrak{h}_{\mathbb{R}}$ denote a real form of $\mathfrak{h}$, and $\mathfrak{h}_{\mathbb{R}}^{*}$ its full dual space. Let $\mathbb{P}$ be the set of all piecewiselinear continuous maps $\pi:[0,1] \longrightarrow \mathfrak{h}_{\mathbb{R}}^{*}$ such that $\pi(0)=0$ and $\pi(1) \in P$, where we set $[0,1]:=\{t \in \mathbb{R} \mid 0 \leq t \leq 1\}$. Also, we set $H_{i}^{\pi}(t):=\alpha_{i}^{\vee}(\pi(t))$ for $t \in[0,1]$, and then $m_{i}^{\pi}:=\min \left\{H_{i}^{\pi}(t) \mid H_{i}^{\pi}(t) \in \mathbb{Z}, t \in[0,1]\right\}$. 
Now we define the root operators $e_{i}, f_{i}: \mathbb{P} \longrightarrow \mathbb{P} \sqcup\{\mathbf{0}\}$ for $i \in I$. First, we set $f_{+}^{i}(\pi):=$ $\max \left\{t \in[0,1] \mid H_{i}^{\pi}(t)=m_{i}^{\pi}\right\}$. If $f_{+}^{i}(\pi)<1$, then we can define $f_{-}^{i}(\pi):=\min \left\{t \in\left[f_{i}^{+}(\pi), 1\right] \mid\right.$ $\left.H_{i}^{\pi}(t)=m_{i}^{\pi}+1\right\}$. In this case, we set

$$
\left(f_{i} \pi\right)(t):= \begin{cases}\pi(t) & t \in\left[0, f_{+}^{i}(\pi)\right], \\ \pi\left(f_{+}^{i}(\pi)\right)+r_{i}\left(\pi(t)-\pi\left(f_{+}^{i}(\pi)\right)\right) & t \in\left[f_{+}^{i}(\pi), f_{-}^{i}(\pi)\right] \\ \pi(t)-\alpha_{i} & t \in\left[f_{-}^{i}(\pi), 1\right] .\end{cases}
$$

Otherwise (i.e., if $f_{+}^{i}(\pi)=1$ ), we set $f_{i} \pi:=\mathbf{0}$.

Next, we define the operator $e_{i}$ for $i \in I^{r e}$. Set $e_{+}^{i}(\pi):=\min \left\{t \in[0,1] \mid H_{i}^{\pi}(t)=m_{i}^{\pi}\right\}$. If $e_{+}^{i}(\pi)>0$, then we can define $e_{-}^{i}(\pi):=\max \left\{t \in\left[0, e_{+}^{i}(\pi)\right] \mid H_{i}^{\pi}(t)=m_{i}^{\pi}+1\right\}$. In this case, we set

$$
\left(e_{i} \pi\right)(t):= \begin{cases}\pi(t) & t \in\left[0, e_{-}^{i}(\pi)\right], \\ \pi\left(e_{-}^{i}(\pi)\right)+r_{i}\left(\pi(t)-\pi\left(e_{-}^{i}(\pi)\right)\right) & t \in\left[e_{-}^{i}(\pi), e_{+}^{i}(\pi)\right] \\ \pi(t)+\alpha_{i} & t \in\left[e_{+}^{i}(\pi), 1\right] .\end{cases}
$$

Otherwise (i.e., if $e_{+}^{i}(\pi)=0$ ), we set $e_{i} \pi:=\mathbf{0}$.

Finally, we define the operator $e_{i}$ for $i \in I^{i m}$. Set $e_{-}^{i}(\pi):=f_{+}^{i}(\pi)$. If $e_{-}^{i}(\pi)<1$ and there exists $t \in\left[e_{-}^{i}(\pi), 1\right]$ such that $H_{i}^{\pi}(t) \geq m_{i}^{\pi}+1-a_{i i}$, then we can define $e_{+}^{i}(\pi):=\min \{t \in$ $\left.\left[e_{-}^{i}(\pi), 1\right] \mid H_{i}^{\pi}(t)=m_{i}^{\pi}+1-a_{i i}\right\}$. We set $e_{i} \pi:=\mathbf{0}$ if $e_{-}^{i}(\pi)=1$, or $e_{-}^{i}(\pi)<1$ and $H_{i}^{\pi}(t)<$ $m_{i}^{\pi}+1-a_{i i}$ for all $t \in\left[e_{-}^{i}(\pi), 1\right]$, or $e_{-}^{i}(\pi)<1$ and there exists $t \in\left[e_{-}^{i}(\pi), 1\right]$ such that $H_{i}^{\pi}(t) \geq m_{i}^{\pi}+1-a_{i i}$ and $H_{i}^{\pi}(s) \leq m_{i}^{\pi}-a_{i i}$ for some $s \in\left[e_{+}^{i}(\pi), 1\right]$. Otherwise, we set

$$
\left(e_{i} \pi\right)(t):= \begin{cases}\pi(t) & t \in\left[0, e_{-}^{i}(\pi)\right] \\ \pi\left(e_{-}^{i}(\pi)\right)+r_{i}^{-1}\left(\pi(t)-\pi\left(e_{-}^{i}(\pi)\right)\right) & t \in\left[e_{-}^{i}(\pi), e_{+}^{i}(\pi)\right] \\ \pi(t)+\alpha_{i} & t \in\left[e_{+}^{i}(\pi), 1\right]\end{cases}
$$

Let $\lambda \in P^{+}$. We write $\mu \geq \nu$ for $\mu, \nu \in \mathcal{W} \lambda:=\{w \lambda \in P \mid w \in \mathcal{W}\}$ if there exists a sequence of elements $\mu=: \lambda_{0}, \lambda_{1}, \ldots, \lambda_{s-1}, \lambda_{s}:=\nu$ in $\mathcal{W} \lambda$ and positive $\operatorname{roots} \beta_{1}, \ldots, \beta_{s} \in \Delta_{r e}^{+} \sqcup \Delta_{i m}$ such that $\lambda_{i-1}=r_{\beta_{i}} \lambda_{i}$ and $\beta_{i}^{\vee}\left(\lambda_{i}\right)>0$ for $i=1, \ldots, s$. This relation $\geq$ on $\mathcal{W} \lambda$ defines a partial order. For $\mu, \nu \in \mathcal{W} \lambda$ and $\beta \in \Delta_{r e}^{+} \sqcup \Delta_{i m}$, we write $\mu \stackrel{\beta}{\leftarrow} \nu$ if $\mu=r_{\beta} \nu, \beta^{\vee}(\nu)>0$, and $\mu$ covers $\nu$ by this partial order. Note that the direction of the arrow $\stackrel{\beta}{\leftarrow}$ defined above is opposite to that in [JL, §5.1.1].

Definition 2.4.1 ([JL, §5.2.1]). For a rational number $a \in(0,1]$ and $\mu, \nu \in \mathcal{W} \lambda$ with $\mu \geq \nu$, an a-chain for $(\mu, \nu)$ is a sequence $\mu=: \nu_{0} \stackrel{\beta_{1}}{\longleftarrow} \nu_{1} \stackrel{\beta_{2}}{\longleftarrow} \cdots \stackrel{\beta_{s}}{\longleftarrow} \nu_{s}:=\nu$ of elements in $\mathcal{W} \lambda$ such that for each $i=1,2, \ldots, s, a \beta_{i}^{\vee}\left(\nu_{i}\right) \in \mathbb{Z}_{>0}$ if $\beta_{i} \in \Delta_{\text {re }}^{+}$, and $a \beta_{i}^{\vee}\left(\nu_{i}\right)=1$ if $\beta_{i} \in \Delta_{i m}$.

Definition 2.4.2 ([JL, §5.2.2]). Let $\boldsymbol{\lambda}:=\left(\lambda_{1}>\lambda_{2}>\cdots>\lambda_{s}\right)$ be a sequence of elements in $\mathcal{W} \lambda$, and $\boldsymbol{a}:=\left(0=a_{0}<a_{1}<\cdots<a_{s}=1\right)$ a sequence of rational numbers. Then, the pair 
$\pi:=(\boldsymbol{\lambda} ; \boldsymbol{a})$ is called a generalized Lakshmibai-Seshadri path (GLS path for short) of shape $\lambda$ if it satisfies the following conditions (called the chain condition): (i) there exists an $a_{i}$-chain for $\left(\lambda_{i}, \lambda_{i+1}\right)$ for each $i=1,2, \ldots, s-1$; (ii) there exists a 1 -chain for $\left(\lambda_{s}, \lambda\right)$.

In this paper, we think of the pair $\pi=(\boldsymbol{\lambda} ; \boldsymbol{a})$ as a path belonging to $\mathbb{P}$ by $\pi(t):=\sum_{i=1}^{j-1}\left(a_{i}-\right.$ $\left.a_{i-1}\right) \lambda_{i}+\left(t-a_{j-1}\right) \lambda_{j}$ for $a_{j-1} \leq t \leq a_{j}$ and $j=1,2, \ldots, s$. We denote by $\mathbb{B}(\lambda)$ the set of all GLS paths of shape $\lambda$.

Now, we define a crystal structure on $\mathbb{B}(\lambda)$. Let $\pi \in \mathbb{B}(\lambda)$. We set $\operatorname{wt}(\pi):=\pi(1) \in P$. For each $i \in I^{r e}$, we set $\varepsilon_{i}(\pi):=-m_{i}^{\pi}, \varphi_{i}(\pi):=\alpha_{i}^{\vee}(\operatorname{wt}(\pi))-m_{i}^{\pi}=H_{i}^{\pi}(1)-m_{i}^{\pi}$. Then, we have $\varepsilon_{i}(\pi)=\max \left\{n \in \mathbb{Z}_{\geq 0} \mid e_{i}^{n} \pi \in \mathbb{P}\right\}$, and $\varphi_{i}(\pi)=\max \left\{n \in \mathbb{Z}_{\geq 0} \mid f_{i}^{n} \pi \in \mathbb{P}\right\}$. For each $i \in I^{i m}$, we set $\varepsilon_{i}(\pi):=0, \varphi_{i}(\pi):=\alpha_{i}^{\vee}(\operatorname{wt}(\pi))$. By the definitions, we have $\varphi_{i}(\pi)=\varepsilon_{i}(\pi)+\alpha_{i}^{\vee}(\operatorname{wt}(\pi))$ for all $i \in I$. Next, we define the Kashiwara operators on $\mathbb{B}(\lambda)$. We use the root operators $e_{i}, i \in I^{\text {re }}$, and $f_{i}, i \in I$, on $\mathbb{P}$ as Kashiwara operators. For $e_{i}, i \in I^{i m}$, we use the "cutoff" of the root operators $e_{i}, i \in I^{i m}$, on $\mathbb{P}(\supset \mathbb{B}(\lambda))$, that is, if $e_{i} \pi \notin \mathbb{B}(\lambda)$, then we set $e_{i} \pi:=\mathbf{0}$ in $\mathbb{B}(\lambda)$ even if $e_{i} \pi \neq \mathbf{0}$ in $\mathbb{P}$. Thus, $\mathbb{B}(\lambda)$ is endowed with a crystal structure. From [JL, Proposition 6.3.5], we have $\mathbb{B}(\lambda)=\mathcal{F} \pi_{\lambda} \backslash\{\mathbf{0}\}$, where $\mathcal{F}$ is the monoid generated by the Kashiwara operators $f_{i}, i \in I$.

\subsection{Embedding of path models}

In this subsection, we give a brief review of the construction of an embedding of JosephLamprou's path model for a given generalized Kac-Moody algebra $\mathfrak{g}$ into Littelmann's path model for an associated Kac-Moody algebra $\tilde{\mathfrak{g}}$. For more details, we refer the reader to [I, §4].

Let $\left(A, \Pi, \Pi^{\vee}, P, P^{\vee}\right)$ be a Borcherds-Cartan datum, and $\left(\widetilde{A}, \widetilde{\Pi}, \widetilde{\Pi} \vee, \widetilde{P}, \widetilde{P}^{\vee}\right)$ be an associated Cartan datum as in $\S 2.2$. For each $\mu \in \mathcal{W} P^{+}=\mathcal{W}_{\text {re }} P^{+}$, we take (and fix) an element $\tilde{\mu} \in \widetilde{P}$ such that

$$
\tilde{\alpha}_{(i, m)}^{\vee}(\tilde{\mu})=\alpha_{i}^{\vee}(\mu) \text { for all }(i, m) \in \tilde{I} .
$$

Let $\widetilde{\mathbb{B}}(\tilde{\mu})$ be the set of all $(\mathrm{G})$ LS paths of shape $\tilde{\mu}$ for $\tilde{\mathfrak{g}}$, and $\widetilde{\mathcal{F}}$ the monoid generated by the Kashiwara operators $f_{(i, m)},(i, m) \in \tilde{I}$. Following the notation of $\S 2.2$, we write $F_{\mathbf{i}}=f_{i_{k}} \cdots f_{i_{2}} f_{i_{1}} \in \mathcal{F}$ for $\mathbf{i}=\left(i_{k}, \ldots, i_{2}, i_{1}\right) \in \mathcal{I}$, and $F_{(\mathbf{i}, \mathbf{m})}=f_{\left(i_{k}, m_{k}\right)} \cdots f_{\left(i_{2}, m_{2}\right)} f_{\left(i_{1}, m_{1}\right)} \in \widetilde{\mathcal{F}}$ for $(\mathbf{i}, \mathbf{m})=\left(\left(i_{s}, m_{s}\right)\right)_{s=1}^{k} \in$ $\widetilde{\mathcal{I}}$.

Proposition 2.5.1 ([I, Proposition 4.1.2]). For a dominant integral weight $\lambda \in P^{+}$, the map

$$
\sim: \mathbb{B}(\lambda) \longrightarrow \widetilde{\mathbb{B}}(\tilde{\lambda}), \pi=F_{\mathbf{i}} \pi_{\lambda} \longmapsto \tilde{\pi}:=F_{(\mathbf{i}, \mathbf{m})} \pi_{\tilde{\lambda}}
$$

is well-defined and injective, where the $\mathbf{m}$ for which $(\mathbf{i}, \mathbf{m}) \in \widetilde{\mathcal{I}}_{\text {ord }}$ is determined uniquely by $\mathbf{i} \in \mathcal{I}$. 
Recall that $B(\lambda)$ denotes the crystal basis of the irreducible highest weight $U_{q}(\mathfrak{g})$-module $V(\lambda)$ of highest weight $\lambda \in P^{+}$. Since we know from $[\mathbf{I}$, Theorem 6.1.1] that $\mathbb{B}(\lambda) \cong B(\lambda)$ as crystals, we obtain the following embedding by Proposition 2.5.1;

$$
\sim: B(\lambda) \hookrightarrow \widetilde{B}(\tilde{\lambda}), b=\widetilde{F}_{\mathbf{i}} u_{\lambda} \mapsto \tilde{b}:=\widetilde{F}_{(\mathbf{i}, \mathbf{m})} \tilde{u}_{\tilde{\lambda}} \text {, for }(\mathbf{i}, \mathbf{m}) \in \widetilde{\mathcal{I}}_{\text {ord }}
$$

where $\widetilde{B}(\tilde{\lambda})$ denotes the crystal basis of the irreducible highest weight $U_{q}(\tilde{\mathfrak{g}})$-module $\widetilde{V}(\tilde{\lambda})$ of highest weight $\tilde{\lambda} \in \widetilde{P}^{+}, \widetilde{F}_{\mathbf{i}}:=\tilde{f}_{i_{k}} \cdots \tilde{f}_{i_{2}} \tilde{f}_{i_{1}}, \widetilde{F}_{(\mathbf{i}, \mathbf{m})}:=\tilde{f}_{\left(i_{k}, m_{k}\right)} \cdots \tilde{f}_{\left(i_{2}, m_{2}\right)} \tilde{f}_{\left(i_{1}, m_{1}\right)}$ are monomials of the Kashiwara operators, and $u_{\lambda} \in V(\lambda), \tilde{u}_{\tilde{\lambda}} \in \widetilde{V}(\tilde{\lambda})$ are the highest weight vectors.

\section{Some representation-theoretical results}

\subsection{Decomposition rules for $U_{q}(\mathfrak{g})$-modules in the category $\mathcal{O}_{\text {int }}$}

In this subsection, we show the decomposition rules for $U_{q}(\mathfrak{g})$-modules stated in Theorems 1 and 2. For this purpose, we recall the following decomposition rules for path crystals.

Theorem 3.1.1 ([I, Theorem 7.1.3]). Let $\lambda, \mu \in P^{+}$. Then, we have an isomorphism of crystals:

$$
\mathbb{B}(\lambda) \otimes \mathbb{B}(\mu) \cong \bigsqcup_{\substack{\pi \in \mathbb{B}(\mu) \\ \tilde{\pi}: \tilde{\lambda} \text {-dominant }}} \mathbb{B}(\lambda+\pi(1)) .
$$

Here, $\tilde{\pi} \in \widetilde{\mathbb{B}}(\tilde{\mu})$ denotes the image of $\pi \in \mathbb{B}(\mu)$ under the embedding $\mathbb{B}(\mu) \hookrightarrow \widetilde{\mathbb{B}}(\tilde{\mu})$, and it is said to be $\tilde{\lambda}$-dominant if $\tilde{\pi}(t)+\tilde{\lambda}$ belongs to the dominant Weyl chamber of $\tilde{\mathfrak{g}}$ for all $t \in[0,1]$.

Let $S \subset I$ be a subset. We set $S^{r e}:=S \cap I^{r e}$ and $S^{i m}:=S \cap I^{i m}$. Also, we set $\widetilde{S}:=$ $\{(i, 1)\}_{i \in S^{r e}} \sqcup\{(i, m)\}_{i \in S^{i m}, m \in \mathbb{Z}_{\geq 1}}$. Let us denote by $\mathfrak{g}_{S}$ (resp., $\tilde{\mathfrak{g}}_{\widetilde{S}}$ ) the Levi subalgebra of $\mathfrak{g}$ corresponding to $S$ (resp., the Levi subalgebra of $\tilde{\mathfrak{g}}$ corresponding to $\widetilde{S}$ ), and denote by $\mathbb{B}_{S}(\lambda)$ the set of all GLS paths of shape $\lambda$ for $\mathfrak{g}_{S}$.

Theorem 3.1.2 ([I, Theorem 7.2.2]). Let $\lambda \in P^{+}$. Then, we have an isomorphism of $\mathfrak{g}_{S^{-}}$ crystals:

$$
\mathbb{B}(\lambda) \cong \bigsqcup_{\substack{\pi \in \mathbb{B}(\lambda) \\ \tilde{\pi}: \tilde{\mathfrak{g}}_{\widetilde{S}} \text {-dominant }}} \mathbb{B}_{S}(\pi(1))
$$

Here, $\tilde{\pi} \in \widetilde{\mathbb{B}}(\tilde{\lambda})$ denotes the image of $\pi \in \mathbb{B}(\lambda)$ under the embedding $\mathbb{B}(\lambda) \hookrightarrow \widetilde{\mathbb{B}}(\tilde{\lambda})$, and it is said to be $\tilde{\mathfrak{g}}_{\widetilde{S}^{-}}$dominant if $\tilde{\pi}(t)$ belongs to the dominant Weyl chamber of $\tilde{\mathfrak{g}}_{\widetilde{S}}$ for all $t \in[0,1]$.

From [JKK, Theorems 3.7 and 7.1], we know the existence and uniqueness of the crystal basis of $U_{q}(\mathfrak{g})$-modules in the category $\mathcal{O}_{\text {int }}$, and the complete reducibility for $U_{q}(\mathfrak{g})$-modules in the category $\mathcal{O}_{\text {int }}\left(\right.$ see $\left[\mathbf{J K K}\right.$, Definition 3.1] for the definition of the category $\mathcal{O}_{\text {int }}$ ). Since 
$V(\lambda) \otimes V(\mu), \lambda, \mu \in P^{+}$, belongs to $\mathcal{O}_{\text {int }}$ for $\mathfrak{g}$, and $V(\lambda)$ belongs to $\mathcal{O}_{\text {int }}$ for $\mathfrak{g}_{S}$ (as a $U_{q}\left(\mathfrak{g}_{S}\right)$ module), Theorems 1 and 2 follow immediately from Theorems 3.1 .1 and 3.1 .2 .

\subsection{Analog of the Parthasarathy-Ranga Rao-Varadarajan conjec- ture for generalized Kac-Moody algebras}

In this subsection, we prove an analog of the Parthasarathy-Ranga Rao-Varadarajan conjecture for generalized Kac-Moody algebras.

In what follows, we denote by $[\mu]$ the unique element in $\mathcal{W}_{\text {re }} \mu \cap P^{+}$for $\mu \in \mathcal{W} P^{+}$. By an argument similar to the one for [Li1, Proposition 7.1], we can show the following lemma.

Lemma 3.2.1 Let $\lambda, \mu \in P^{+}$, and $\pi:=\left(\mu_{1}, \ldots, \mu_{l} ; a_{0}, a_{1}, \ldots, a_{l}\right) \in \mathbb{B}(\mu)$. If $\lambda+\pi\left(a_{p}\right) \in P^{+}$for all $0 \leq p \leq l-1$, then there exists a $\lambda$-dominant path $\pi^{\prime} \in \mathbb{B}(\mu)$ such that $\lambda+\pi^{\prime}(1)=[\lambda+\pi(1)]$.

Let $r_{(i, m)},(i, m) \in \tilde{I}$, denote the simple reflection of the Weyl group $\widetilde{W}$ of $\tilde{\mathfrak{g}}, \widetilde{W}_{r e} \subset \widetilde{W}$ the subgroup of $\widetilde{W}$ generated by $r_{(i, 1)}, i \in I^{r e}$, and $\widetilde{W}_{\text {gen }}:=\left\{R_{(\mathbf{i}, \mathbf{m})} \in \widetilde{W} \mid(\mathbf{i}, \mathbf{m}) \in \widetilde{\mathcal{I}}_{\text {gen }}\right\}$, where $R_{(\mathbf{i}, \mathbf{m})}:=r_{\left(i_{l}, m_{l}\right)} \cdots r_{\left(i_{1}, m_{1}\right)}$ for $(\mathbf{i}, \mathbf{m})=\left(\left(i_{s}, m_{s}\right)\right)_{s=1}^{l} \in \widetilde{\mathcal{I}}$. Note that $\widetilde{W}_{\text {gen }}$ is not closed under multiplication, and that $\widetilde{W}_{r e}$ is isomorphic as a group to the submonoid $\mathcal{W}_{\text {re }}$ of $\mathcal{W}$; we write this isomorphism as

$$
\sim: \mathcal{W}_{r e} \stackrel{\cong}{\longrightarrow} \widetilde{W}_{r e}, w=r_{i_{l}} \cdots r_{i_{1}} \longmapsto \tilde{w}:=r_{\left(i_{l}, 1\right)} \cdots r_{\left(i_{1}, 1\right)} \text {, for } i_{1}, \ldots, i_{l} \in I^{r e}
$$

Also, we can easily check that the map $\widetilde{W}_{\text {gen }} \rightarrow \mathrm{GL}\left(\mathfrak{h}^{*}\right), R_{(\mathbf{i}, \mathbf{m})} \mapsto R_{\mathbf{i}}$, is well-defined, where $R_{\mathbf{i}}:=r_{i_{l}} \cdots r_{i_{1}}$ for $\mathbf{i}=\left(i_{l}, \ldots, i_{1}\right) \in \mathcal{I}$.

Lemma 3.2.2 Let $\lambda \in P^{+}$. If $F_{(\mathbf{i}, \mathbf{m})} \pi_{\tilde{\lambda}},(\mathbf{i}, \mathbf{m}) \in \widetilde{\mathcal{I}}_{\text {gen }}$, is not $\mathbf{0}$ in $\widetilde{\mathbb{B}}(\tilde{\lambda})$, then $F_{(\mathbf{i}, \overline{\mathbf{m}})} \pi_{\tilde{\lambda}}$ is not $\mathbf{0}$ in $\widetilde{\mathbb{B}}(\tilde{\lambda})$, where the element $(\mathbf{i}, \overline{\mathbf{m}}) \in \widetilde{\mathcal{I}}_{\text {ord }}$ is determined uniquely by $\mathbf{i} \in \mathcal{I}$.

Proof. Since $(\mathbf{i}, \mathbf{m}) \in \widetilde{\mathcal{I}}_{\text {gen }}$, there exists a permutation $\omega \in \Omega$ on $\widetilde{\mathcal{I}}$ that sends $(\mathbf{i}, \mathbf{m})$ to $(\mathbf{i}, \overline{\mathbf{m}})$ (see $\S 2.2$ ). Hence we have a permutation on the path crystal $\widetilde{\mathbb{B}}(\tilde{\lambda})$, induced by the diagram automorphism of $\tilde{\mathfrak{g}}$ corresponding to the $\omega \in \Omega$, that sends $F_{(\mathbf{i}, \mathbf{m})} \pi_{\tilde{\lambda}}$ to $F_{(\mathbf{i}, \overline{\mathbf{m}})} \pi_{\tilde{\lambda}}$ (see [NS, Lemma 3.1.1]). Therefore, we see that $F_{(\mathbf{i}, \overline{\mathbf{m}})} \pi_{\tilde{\lambda}} \neq \mathbf{0}$.

For a path $\eta=F_{(\mathbf{i}, \mathbf{m})} \pi_{\tilde{\lambda}} \in \widetilde{\mathbb{B}}(\tilde{\lambda})$ with $(\mathbf{i}, \mathbf{m}) \in \widetilde{\mathcal{I}}_{\text {gen }}$, we set $\bar{\eta}:=F_{(\mathbf{i}, \overline{\mathbf{m}})} \pi_{\tilde{\lambda}}$, with $(\mathbf{i}, \overline{\mathbf{m}}) \in \widetilde{\mathcal{I}}_{\text {ord }}$. From the proof of Lemma 3.2.2, $\bar{\eta}$ is independent of the choice of an expression for $\eta$ of the form $F_{(\mathbf{i}, \mathbf{m})} \pi_{\tilde{\lambda}}$ with $(\mathbf{i}, \mathbf{m}) \in \widetilde{\mathcal{I}}_{\text {gen }}$. The following lemma is obvious.

Lemma 3.2.3 Let $\eta \in \widetilde{\mathbb{B}}(\tilde{\lambda})$, and write $\eta(1)$ as $\tilde{\lambda}-\sum c_{(i, m)} \tilde{\alpha}_{(i, m)}, c_{(i, m)} \in \mathbb{Z}_{\geq 0}$. If $c_{(i, m)} \in\{0,1\}$ for all $i \in I^{i m}$ and $m \in \mathbb{Z}_{\geq 1}$, then there exists a path $\pi \in \mathbb{B}(\lambda)$ such that $\tilde{\pi}=\bar{\eta}$ in $\widetilde{\mathbb{B}}(\tilde{\lambda})$. 
For $\mathbf{i}=\left(i_{k}, i_{k-1}, \ldots, i_{1}\right) \in \mathcal{I}$, we set $\mathbf{i}_{[s]}:=\left(i_{s}, i_{s-1}, \ldots, i_{1}\right), 1 \leq s \leq k$. Let $\lambda, \mu \in P^{+}$ and $R_{\mathbf{i}}, R_{\mathbf{j}} \in \mathcal{W}, \mathbf{i}=\left(i_{k}, \ldots, i_{2}, i_{1}\right), \mathbf{j}=\left(j_{l}, \ldots, j_{2}, j_{1}\right) \in \mathcal{I}$, be such that $\alpha_{i_{s}}^{\vee}\left(R_{\mathbf{i}_{[s-1]}} \lambda\right)=$ 1 (resp., $\left.\alpha_{j_{t}}^{\vee}\left(R_{\mathbf{j}_{[t-1]}} \mu\right)=1\right)$ if $i_{s} \in I^{i m}$ (resp., $\left.j_{t} \in I^{i m}\right)$. We take sequences $\mathbf{m}=\left(m_{k}, \ldots, m_{2}, m_{1}\right)$ and $\mathbf{n}=\left(n_{l}, \ldots, n_{2}, n_{1}\right)$ of positive integers such that $(\mathbf{i}, \mathbf{m}),(\mathbf{j}, \mathbf{n}) \in \widetilde{\mathcal{I}}_{\text {gen }}$, and such that $m_{s} \neq n_{t}$ if $i_{s}=j_{t}$ in $I^{i m}$. Also, we set $\nu:=R_{\mathbf{i}} \lambda+R_{\mathbf{j}} \mu \in P$ and $\bar{\nu}:=R_{(\mathbf{i}, \mathbf{m})} \tilde{\lambda}+R_{(\mathbf{j}, \mathbf{n})} \tilde{\mu} \in \widetilde{P}$; note that $\bar{\nu} \neq \tilde{\nu}$ in general, where $\tilde{\nu} \in \widetilde{P}$ is defined by equation (1) in $\S 2.5$ for a weight $\nu \in \mathcal{W} P^{+}$. With this notation, we have the following.

Theorem 3.2.4 If $\bar{\nu} \in \widetilde{P}^{+}$, then $V(\nu)$ appears in the irreducible decomposition of $V(\lambda) \otimes V(\mu)$.

Proof. If we take $M \in \mathbb{Z}_{\geq 1}$ such that $(i, M)$ does not appear in $(\mathbf{i}, \mathbf{m})$ and $(\mathbf{j}, \mathbf{n})$ for any $i \in I$, then the equality $\alpha_{i}^{\vee}(\nu)=\tilde{\alpha}_{(i, M)}^{\vee}(\bar{\nu})$ holds for all $i \in I$ by the definition of $\bar{\nu}$. Since $\tilde{\alpha}_{(i, M)}^{\vee}(\bar{\nu}) \geq 0$, we deduce that $\nu$ is a dominant integral weight. By Theorem 1, it suffices to show that there exists $\pi \in \mathbb{B}(\mu)$ such that $\tilde{\pi} \in \widetilde{\mathbb{B}}(\tilde{\mu})$ is $\tilde{\lambda}$-dominant and $\lambda+\pi(1)=\nu$. Set $\eta_{1}:=\left(R_{(\mathbf{i}, \mathbf{m})}^{-1} R_{(\mathbf{j}, \mathbf{n})} \tilde{\mu} ; 0,1\right) \in \widetilde{\mathbb{B}}(\tilde{\mu})$, with $R_{(\mathbf{i}, \mathbf{m})}, R_{(\mathbf{j}, \mathbf{n})} \in \widetilde{W}_{\text {gen }}$. By Lemma 3.2.1, there exists a $\tilde{\lambda}$-dominant path $\eta_{2} \in \widetilde{\mathbb{B}}(\tilde{\mu})$ such that $\tilde{\lambda}+\eta_{2}(1)=\left[\tilde{\lambda}+\eta_{1}(1)\right]=\bar{\nu}$. If we write $\eta_{2}(1)$ as $\tilde{\mu}-\sum c_{(i, m)} \tilde{\alpha}_{(i, m)}$, then $c_{(i, m)} \in\{0,1\}$ for all $i \in I^{i m}$ and $m \in \mathbb{Z}_{\geq 1}$ by the definition of $\bar{\nu}$. Therefore, by Lemma 3.2.3, there exists $\pi \in \mathbb{B}(\mu)$ such that $\tilde{\pi}=\bar{\eta}_{2}$. From this, we conclude that $\tilde{\pi} \in \widetilde{\mathbb{B}}(\tilde{\mu})$ is $\tilde{\lambda}$-dominant and $\lambda+\pi(1)=\nu$.

Corollary 3.2.5 Let $\lambda, \mu \in P^{+}, w_{1}, w_{2} \in \mathcal{W}_{\text {re }}$, and set $\nu=w_{1} \lambda+w_{2} \mu$. If $\nu \in P^{+}$, then $V(\nu)$ appears in the irreducible decomposition of $V(\lambda) \otimes V(\mu)$.

Remark 3.2.6 For general elements $w_{1}, w_{2}$ in the monoid $\mathcal{W}$, the statement of the corollary above is false. To see this, we take $\lambda, \mu \in P^{+}$and $i \in I^{\text {im }}$ such that $\alpha_{i}^{\vee}(\lambda)=0$ and $\alpha_{i}^{\vee}(\mu)=1$. Then, $\nu:=\lambda+r_{i} \mu=\lambda+\mu-\alpha_{i}$ is a dominant integral weight for $\mathfrak{g}$. However, we have $(\mathbb{B}(\lambda) \otimes \mathbb{B}(\mu))_{\nu}=\left\{\pi_{\lambda} \otimes f_{i} \pi_{\mu}\right\}$, and $e_{i}\left(\pi_{\lambda} \otimes f_{i} \pi_{\mu}\right)=\pi_{\lambda} \otimes e_{i} f_{i} \pi_{\mu}=\pi_{\lambda} \otimes \pi_{\mu} \neq \mathbf{0}$. Therefore, there is no highest weight vector of weight $\nu$, and $V(\nu)$ cannot be an irreducible component of $V(\lambda) \otimes V(\mu)$. As for $\tilde{\mathfrak{g}}$, we have $\bar{\nu}:=\tilde{\lambda}+r_{(i, 1)} \tilde{\mu}, \tilde{\alpha}_{(i, 1)}^{\vee}(\bar{\nu})=\tilde{\alpha}_{(i, 1)}^{\vee}(\tilde{\lambda})+\tilde{\alpha}_{(i, 1)}^{\vee}\left(r_{(i, 1)} \tilde{\mu}\right)=\alpha_{i}^{\vee}(\lambda)-\alpha_{i}^{\vee}(\mu)=-1$, and hence we see that $\bar{\nu}$ is not a dominant integral weight for $\tilde{\mathfrak{g}}$.

\section{Demazure modules for generalized Kac-Moody alge- bras}

\subsection{Definition of Demazure modules}

Let $\lambda \in P^{+}$and $w=v_{l} r_{j_{l}} \cdots v_{1} r_{j_{1}} v_{0} \in \mathcal{W}$, with $j_{1}, \ldots, j_{l} \in I^{i m}, v_{0}, \ldots, v_{l} \in \mathcal{W}_{r e}$, be such that

$$
\alpha_{j_{s}}^{\vee}\left(v_{s-1} r_{j_{s-1}} \cdots v_{1} r_{j_{1}} v_{0}(\lambda)\right)=1 \text { for all } s=1,2, \ldots, l \text {. }
$$


Note that the condition (44) for $w$ and $\lambda$ is independent of the choice of an expression for $w$ since this condition is preserved under the relations (1), (2), and (3) of Definition 2.3.1. For such an element $w \in \mathcal{W}$, we set $V_{w}(\lambda):=U_{q}^{+}(\mathfrak{g}) V(\lambda)_{w \lambda}$, where $V(\lambda)_{\mu} \subset V(\lambda)$ denotes the weight space of weight $\mu \in P$. We call $V_{w}(\lambda)$ the Demazure (sub) module of $V(\lambda)$ of lowest weight $w \lambda$. Since $V_{w}(\lambda)$ depends only on the weight $w \lambda \in P$, we may assume that the element $w=R_{\mathbf{i}}$, with $\mathbf{i}=\left(i_{k}, \ldots, i_{2}, i_{1}\right)$, satisfies the following condition (recall the notation $\mathbf{i}_{[t]}=\left(i_{t}, \ldots, i_{2}, i_{1}\right), t=$ $1, \ldots, k)$ :

$$
\alpha_{i_{t}}^{\vee}\left(R_{\mathbf{i}_{[t-1]}} \lambda\right)>0 \text { for all } t=1,2, \ldots, k \text {. }
$$

We will show that the Demazure module $V_{w}(\lambda)$ is generated by a single weight vector. Namely, we will show that $\operatorname{dim} V(\lambda)_{w \lambda}=1$; note that the action of $\mathcal{W}$ on the set of weights of $V(\lambda)$ does not necessarily preserve the weight multiplicities. Before doing this, we recall the action of $\mathcal{W}_{\text {re }}$ on $\mathbb{B}(\lambda)$ (see $[\mathbf{J L}, \S 9.2]$ ): for $\pi \in \mathbb{B}(\lambda)$ and $v=r_{i_{k}} \cdots r_{i_{2}} r_{i_{1}} \in \mathcal{W}_{r e}$, with $i_{1}, i_{2}, \ldots, i_{k} \in I^{r e}$, define

$$
S_{v} \pi:=x_{i_{k}}^{\alpha_{i_{k}}^{\vee}\left(R_{\mathrm{i}_{[k-1]}} \mathrm{wt}(\pi)\right)} \cdots x_{i_{2}}^{\alpha_{i_{2}}^{\vee}\left(R_{\mathbf{i}_{[1]}} \mathrm{wt}(\pi)\right)} x_{i_{1}}^{\alpha_{i_{1}}^{\vee}(\mathrm{wt}(\pi))} \pi
$$

where $x_{i}^{a}:=f_{i}^{a}$ if $a \geq 0, x_{i}^{a}:=e_{i}^{-a}$ if $a<0$, and $\mathbf{i}=\left(i_{k}, \ldots, i_{2}, i_{1}\right)$. It is obvious that $\operatorname{wt}\left(S_{v} \pi\right)=v(\operatorname{wt}(\pi))$.

Lemma 4.1.1 If $w \in \mathcal{W}$ and $\lambda \in P^{+}$satisfy condition (4), then we have $\operatorname{dim} V(\lambda)_{w \lambda}=1$.

Proof. Let $w=v_{l} r_{j_{l}} \cdots v_{1} r_{j_{1}} v_{0}$, with $v_{0}, \ldots, v_{l} \in \mathcal{W}_{r e}, j_{1}, \ldots, j_{l} \in I^{i m}$, be a reduced expression satisfying condition (44). It suffices to show that $\# \mathbb{B}(\lambda)_{w \lambda}=1$. As remarked above, we may assume further that this expression for $w$ satisfies condition (5) .

If we set $\pi:=S_{v_{l}} f_{j_{l}} \cdots S_{v_{1}} f_{j_{1}} S_{v_{0}} \pi_{\lambda}$, then the $x$ 's in the expression (6) for $S_{v_{s}}, s=0,1, \ldots, l$, are all $f$ by condition (5), and hence $\pi=\pi_{w \lambda}$ by condition (41). Also, by using the fact [I, Lemma 4.1.5], we can deduce that the element $\tilde{\pi} \in \widetilde{\mathbb{B}}(\tilde{\lambda})$ (see Proposition 2.5.1) can be written as $\tilde{\pi}=$ $S_{\tilde{v}_{l}} f_{\left(j_{l}, m_{l}\right)} \cdots S_{\tilde{v}_{1}} f_{\left(j_{1}, m_{1}\right)} S_{\tilde{v}_{0}} \pi_{\tilde{\lambda}}=\pi_{\tilde{w} \tilde{\lambda}}$, where $\left(\left(j_{s}, m_{s}\right)\right)_{s=1}^{l} \in \tilde{\mathcal{I}}_{\text {ord }}, \tilde{w}:=\tilde{v}_{l} r_{\left(j_{l}, m_{l}\right)} \cdots \tilde{v}_{1} r_{\left(j_{1}, m_{1}\right)} \tilde{v}_{0} \in$ $\widetilde{W}$, and each $\tilde{v}_{s} \in \widetilde{W}_{r e}$ corresponds to $v_{s} \in \mathcal{W}_{r e}$ via the isomorphism (3) in $\S 3.2$. This shows that the image of $\mathbb{B}(\lambda)_{w \lambda}$ under the embedding of Proposition 2.5.1 is contained in $\widetilde{\mathbb{B}}(\tilde{\lambda})_{\tilde{w} \tilde{\lambda}}$; note that if $\pi_{1}$ and $\pi_{2}$ in $\mathbb{B}(\lambda)$ satisfy $\operatorname{wt}\left(\pi_{1}\right)=\operatorname{wt}\left(\pi_{2}\right)$, then $\operatorname{wt}\left(\tilde{\pi}_{1}\right)=\operatorname{wt}\left(\tilde{\pi}_{2}\right)$. Since $\widetilde{\mathbb{B}}(\tilde{\lambda})_{\tilde{w} \tilde{\lambda}}=\left\{\pi_{\tilde{w} \tilde{\lambda}}\right\}$, we conclude that $\mathbb{B}(\lambda)_{w \lambda}=\left\{\pi_{w \lambda}\right\}$, and hence $\# \mathbb{B}(\lambda)_{w \lambda}=1$.

Remark 4.1.2 Since the element $\tilde{w} \in \widetilde{W}$ in the proof of Lemma 4.1.1 is a minimal coset representative of a coset in $\widetilde{W} / \widetilde{W}_{\tilde{\lambda}}$, where $\widetilde{W}_{\tilde{\lambda}} \subset \widetilde{W}$ denotes the isotropy subgroup for $\tilde{\lambda} \in \widetilde{P}^{+}$ (see $[\mathbf{H u}, \S 1.10$ and $\S 1.12]$ ), by the uniqueness of minimal coset representative, $\tilde{w}$ is independent of the choice of an expression for $w$ satisfying conditions (4) and (5) for $\lambda \in P^{+}$. 


\subsection{Minimal dominant reduced expressions}

In this subsection, we introduce a specific expression for $w \in \mathcal{W}$, which satisfies conditions (4) and (5) for $\lambda \in P^{+}$, in order to state Theorem 4 (see $\S 1$ ).

Lemma 4.2.1 Let $w \in \mathcal{W}$ satisfy conditions (4) and (5) for $\lambda \in P^{+}$. Then, $w$ has an expression $w=w_{k} r_{i_{k}}^{a_{k}} \cdots w_{1} r_{i_{1}}^{a_{1}} w_{0}$, with $w_{0}, \ldots, w_{k} \in \mathcal{W}_{\text {re }}$ and $a_{1}, \ldots, a_{k} \in \mathbb{Z}_{\geq 1}$, where $i_{1}, \ldots, i_{k} \in I^{i m}$ are all distinct. Moreover, if $s \in\left\{1 \leq t \leq k \mid a_{t}>1\right\}$, then $a_{i_{s}, i_{s}}=0$, i.e., the $\left(i_{s}, i_{s}\right)$-entry of the Borcherds-Cartan matrix $A$ is zero.

Proof. Let $w=v_{l} r_{j_{l}} \cdots v_{1} r_{j_{1}} v_{0}$, with $v_{0}, \ldots, v_{l} \in \mathcal{W}_{r e}, j_{1}, \ldots, j_{l} \in I^{i m}$, be a reduced expression satisfying conditions (44) and (5) for $\lambda \in P^{+}$. If $j_{s}=j_{t}$ with $s>t$, then we have

$$
\alpha_{j_{s}}^{\vee}\left(v_{s-1} r_{j_{s-1}} \cdots v_{t} r_{j_{t}}\left(v_{t-1} r_{j_{t-1}} \cdots v_{1} r_{j_{1}} v_{0}(\lambda)\right)\right)=\alpha_{j_{t}}^{\vee}\left(v_{t-1} r_{j_{t-1}} \cdots v_{1} r_{j_{1}} v_{0}(\lambda)\right)
$$

by condition (4). Also, by condition (5), $r_{j_{s}}$ commutes with $v_{s-1}, \ldots, v_{t+1}, v_{t}$ and $r_{j_{s-1}}, \ldots, r_{j_{t+1}}, r_{j_{t}}$ since $\alpha_{j_{s}}^{\vee}$ is an anti-dominant integral coweight by [JL, Lemma 2.1.11]. In particular, we obtain $a_{j_{s}, j_{s}}=0$.

We fix an expression $w=w_{k} r_{i_{k}}^{a_{k}} \cdots w_{1} r_{i_{1}}^{a_{1}} w_{0}$ given in Lemma 4.2.1 for which the sequence $\left(\ell\left(w_{0}\right), \ell\left(w_{1}\right), \ldots, \ell\left(w_{k}\right)\right)$ is minimal in lexicographic order among all such expressions of $w$. Then, this is a dominant reduced expression (see Definition 2.3.3). We call this expression a minimal dominant reduced expression (with respect to $\lambda \in P^{+}$).

Here we collect some fundamental properties of minimal dominant reduced expression $w=$ $w_{k} r_{i_{k}}^{a_{k}} \cdots w_{1} r_{i_{1}}^{a_{1}} w_{0}$; these properties follow directly from the definition and by induction on $k$ and $\ell(w)$. For each $0 \leq s \leq k$, let $w_{s}=r_{s, \ell_{s}} \cdots r_{s, 2} r_{s, 1}$, with $r_{s, p}:=r_{\alpha_{s, p}}, \alpha_{s, p} \in \Pi_{r e}$, be a (fixed) reduced expression, where $\ell_{s}:=\ell\left(w_{s}\right)$.

Lemma 4.2.2 With the notation above, the following statements hold.

(a) $\alpha_{s, p}^{\vee}\left(r_{s, p-1} \cdots r_{s, 2} r_{s, 1} r_{i_{s-1}}^{a_{s-1}} \cdots w_{1} r_{i_{1}}^{a_{1}} w_{0} \lambda\right)=1$ for all $0 \leq s \leq k-1$ and $1 \leq p \leq \ell_{s}$.

(b) $\alpha_{s, p}^{\vee}\left(\alpha_{s, p-1}\right)=-1$ for all $0 \leq s \leq k-1$ and $1<p \leq \ell_{s}$.

(c) $\alpha_{s, p}^{\vee}\left(\alpha_{t, q}\right)=0$ and $\alpha_{s, p}^{\vee}\left(\alpha_{i_{t}}\right)=0$ if $(t, q)<(s, p-1)$ in lexicographic order.

(d) $\alpha_{i_{s}}^{\vee}\left(\alpha_{s-1,1}\right)=-1$ and $\alpha_{i_{s}}^{\vee}\left(\alpha_{t, q}\right)=0$ if $(t, q)<(s-1,1)$ in lexicographic order.

(e) $\alpha_{0,1}^{\vee}(\lambda)=1$ and $\alpha_{s, p}^{\vee}(\lambda)=0$ for $0 \leq s \leq k-1$ and $1 \leq p \leq \ell_{s}$ such that $(s, p) \neq(0,1)$.

(f) If $s \neq 0$ or $w_{0} \neq 1$, then $\alpha_{i_{s}}^{\vee}(\lambda)=0$ for $0 \leq s \leq k-1$.

(g) If $w_{0}=1$, then $\alpha_{i_{1}}^{\vee}(\lambda)=1$. 


\subsection{Description of Demazure modules}

This subsection is devoted to the proof of Proposition 4.3.1 below. Before doing this, we fix some notation. Let $\mathfrak{g}_{r e} \subset \mathfrak{g}$ be the Levi subalgebra corresponding to $I^{r e}$; note that $\mathfrak{g}_{r e}$ is an ordinary Kac-Moody algebra. For a (fixed) reduced expression $v=r_{j_{l}} \cdots r_{j_{2}} r_{j_{1}} \in \mathcal{W}_{r e}$, with $j_{l}, \ldots, j_{2}, j_{1} \in I^{r e}$, we set $F_{v}^{\mathbf{m}}:=f_{j_{l}}^{m_{l}} \cdots f_{j_{2}}^{m_{2}} f_{j_{1}}^{m_{1}}$ and $\widetilde{F}_{v}^{\mathbf{m}}:=\tilde{f}_{j_{l}}^{m_{l}} \cdots \tilde{f}_{j_{2}}^{m_{2}} \tilde{f}_{j_{1}}^{m_{1}}$. Also, for $\mathbf{i}=\left(i_{l}, \ldots, i_{2}, i_{1}\right)$, set $F_{\mathbf{i}}^{\mathbf{m}}:=f_{i_{l}}^{m_{l}} \cdots f_{i_{2}}^{m_{2}} f_{i_{1}}^{m_{1}}$ and $F_{\mathbf{i}}^{\max }:=f_{i_{l}}^{\max } \cdots f_{i_{1}}^{\max }$, where $f_{j}^{\max } u, j \in I^{r e}$, $u \in V(\lambda)$, denotes the element $f_{j}^{p} u \neq 0, p \geq 0$, such that $f_{j}^{p+1} u=0$. By convention, we write $F_{v}:=F_{v}^{\mathbf{m}}$ if $\mathbf{m}=(1, \ldots, 1,1)$.

Proposition 4.3.1 Let $w \in \mathcal{W}$ satisfy conditions (4) and (5) for $\lambda \in P^{+}$, and fix a minimal dominant reduced expression $w=w_{k} r_{i_{k}}^{a_{k}} \cdots w_{1} r_{i_{1}}^{a_{1}} w_{0}$. Then, with the notation above, we have

(1) $V_{w}(\lambda)=\sum_{\underline{\mathbf{m}}, \underline{\epsilon}} \mathbb{C}(q) F_{w_{k}}^{\mathbf{m}_{k}} f_{i_{k}}^{\epsilon_{k}} \cdots F_{w_{1}}^{\mathbf{m}_{1}} f_{i_{1}}^{\epsilon_{1}} F_{w_{0}}^{\mathbf{m}_{0}} u_{\lambda}$, where the summation is over all $\underline{\mathbf{m}}=\left(\mathbf{m}_{s}\right)_{s=0}^{k} \in$ $\prod_{s=0}^{k} \mathbb{Z}_{\geq 0}^{\ell\left(w_{s}\right)}$ and all $\underline{\boldsymbol{\epsilon}}=\left(\epsilon_{t}\right)_{t=1}^{k}, 0 \leq \epsilon_{t} \leq a_{t}, 1 \leq t \leq k$.

(2) $V_{v}(\lambda) \subset V_{w}(\lambda)$ if $v \leq w$ in $\mathcal{W}$.

Part (2) of Proposition 4.3.1 follows from part (1), and part (1) for $k=1$ is established by Lemmas 4.3 .2 and 4.3 .3 below; part (1) for $k \geq 2$ follows immediately by induction on $k$.

Now, suppose that $k=1$ for the expression of $w$ above, and write $w=R_{\mathbf{i}} r_{i}^{a} R_{\mathbf{j}}$, with $a \in \mathbb{Z}_{\geq 1}$, $i \in I^{i m}, \mathbf{i}=\left(i_{s}, \ldots, i_{2}, i_{1}\right), \mathbf{j}=\left(j_{t}, \ldots, j_{2}, j_{1}\right)$, such that $R_{\mathbf{i}}, R_{\mathbf{j}} \in \mathcal{W}_{r e}$ are reduced expressions. In this case, we have $V_{w}(\lambda)=U_{q}^{+}(\mathfrak{g}) u_{w \lambda}$, where $u_{w \lambda}:=F_{\mathbf{i}}^{\max } f_{i}^{a} F_{\mathbf{j}} u_{\lambda}=F_{\mathbf{i}}^{\max } f_{i}^{a} F_{\mathbf{j}}^{\max } u_{\lambda} \in$ $V(\lambda)_{w \lambda} \backslash\{0\}$ by Lemma 4.2.2. Set $J:=I \backslash\{i\}$, and denote by $\mathfrak{g}_{J} \subset \mathfrak{g}$ the Levi subalgebra corresponding to $J$. If we write $\left(e_{i} U_{q}^{+}\left(\mathfrak{g}_{J}\right)\right)^{m}:=\left(e_{i} U_{q}^{+}\left(\mathfrak{g}_{J}\right)\right) \cdots\left(e_{i} U_{q}^{+}\left(\mathfrak{g}_{J}\right)\right)$ (m times), then $U_{q}^{+}(\mathfrak{g})=\bigoplus_{m=0}^{\infty} U_{q}^{+}\left(\mathfrak{g}_{J}\right)\left(e_{i} U_{q}^{+}\left(\mathfrak{g}_{J}\right)\right)^{m}$ and we have the following by weight consideration:

$$
V_{w}(\lambda)=\bigoplus_{m=0}^{\infty} U_{q}^{+}\left(\mathfrak{g}_{J}\right)\left(e_{i} U_{q}^{+}\left(\mathfrak{g}_{J}\right)\right)^{m} u_{w \lambda}=\bigoplus_{m=0}^{a} U_{q}^{+}\left(\mathfrak{g}_{J}\right)\left(e_{i} U_{q}^{+}\left(\mathfrak{g}_{J}\right)\right)^{m} u_{w \lambda}
$$

where the number $a$ in the right-hand side of (7) is the one appearing in the expression $w=$ $R_{\mathbf{i}} r_{i}^{a} R_{\mathbf{j}}$. By Lemma 4.2.2, we have $f_{i}^{m} F_{\mathbf{j}} u_{\lambda} \in V(\lambda)_{r_{i}^{m} R_{\mathbf{j}} \lambda} \backslash\{0\}$ and $F_{\mathbf{i}}{ }^{\max } f_{i}^{m} F_{\mathbf{j}} u_{\lambda} \in V(\lambda)_{R_{\mathbf{i}} r_{i}^{m} R_{\mathbf{j}} \lambda} \backslash$ $\{0\}, 0 \leq m \leq a$. Note that $r_{i}^{m} R_{\mathbf{j}} \lambda \in P^{+}$for all $m \geq 1$ since $R_{\mathbf{i}} r_{i}^{m} R_{\mathbf{j}}$ is a (minimal) dominant reduced expression. Therefore, each $f_{i}^{m} F_{\mathbf{j}} u_{\lambda}$ for $1 \leq m \leq a$ is a $U_{q}\left(\mathfrak{g}_{r e}\right)$-highest weight vector.

Lemma 4.3.2 For each $1 \leq m \leq a$, we have

$$
U_{q}^{+}\left(\mathfrak{g}_{J}\right)\left(e_{i} U_{q}^{+}\left(\mathfrak{g}_{J}\right)\right)^{a-m} u_{w \lambda}=\sum_{\mathbf{n} \in \mathbb{Z}_{\geq 0}^{s}} \mathbb{C}(q) F_{\mathbf{i}}^{\mathbf{n}} f_{i}^{m} F_{\mathbf{j}} u_{\lambda}
$$


Proof. We proceed by descending induction on $m$. If $m=a$, then we have $e_{j} U_{q}^{+}\left(\mathfrak{g}_{J}\right) u_{w \lambda}=\{0\}$ for all $j \in J \backslash I^{r e}$ by weight consideration. Hence it follows that $U_{q}^{+}\left(\mathfrak{g}_{J}\right) u_{w \lambda}=U_{q}^{+}\left(\mathfrak{g}_{r e}\right) u_{w \lambda}$. Also, $U_{q}^{+}\left(\mathfrak{g}_{r e}\right) u_{w \lambda}$ is identical to $\sum_{\mathbf{n}} \mathbb{C}(q) F_{\mathbf{i}}^{\mathbf{n}} f_{i}^{a} F_{\mathbf{j}} u_{\lambda}$ by $[\mathbf{K a s} 2$, Corollary 3.2.2]. Thus, Lemma 4.3.2 follows in this case.

Suppose that $1 \leq m \leq a-1$. By the induction hypothesis, we have

$$
U_{q}^{+}\left(\mathfrak{g}_{J}\right)\left(e_{i} U_{q}^{+}\left(\mathfrak{g}_{J}\right)\right)^{a-m} u_{w \lambda}=U_{q}^{+}\left(\mathfrak{g}_{J}\right) e_{i} \sum_{\mathbf{n}} \mathbb{C}(q) F_{\mathbf{i}}^{\mathbf{n}} f_{i}^{m+1} F_{\mathbf{j}} u_{\lambda}
$$

which is identical to $U_{q}^{+}\left(\mathfrak{g}_{J}\right) \sum \mathbb{C}(q) F_{\mathbf{i}}^{\mathbf{n}} e_{i} f_{i}^{m+1} F_{\mathbf{j}} u_{\lambda}$ since $i$ does not appear in $\mathbf{i}$ and each $F_{\mathbf{i}}^{\mathbf{n}}$ in the summation commutes with $e_{i}$. Since $a_{i i}=0$ by Lemma 4.2.1, it follows that

$$
e_{i} f_{i}^{m+1} F_{\mathbf{j}} u_{\lambda}=\underbrace{\{m+1\}_{i}}_{=m+1(\geq 2)}[\underbrace{\alpha_{i}^{\vee}\left(\lambda-\alpha_{j_{1}}-\cdots-\alpha_{j_{t}}\right)}_{=1}-\underbrace{\frac{1}{2} a_{i i} m}_{=0}]_{i} f_{i}^{m} F_{\mathbf{j}} u_{\lambda}
$$

by [JKK, Lemma 2.5]. Therefore, we obtain

$$
U_{q}^{+}\left(\mathfrak{g}_{J}\right) \sum \mathbb{C}(q) F_{\mathbf{i}}^{\mathbf{n}} e_{i} f_{i}^{m+1} F_{\mathbf{j}} u_{\lambda}=U_{q}^{+}\left(\mathfrak{g}_{J}\right) \sum \mathbb{C}(q) F_{\mathbf{i}}^{\mathbf{n}} f_{i}^{m} F_{\mathbf{j}} u_{\lambda}
$$

Also, we see that the right-hand side of (8) is identical to $U_{q}^{+}\left(\mathfrak{g}_{r e}\right) \sum \mathbb{C}(q) F_{\mathbf{i}}^{\mathbf{n}} f_{i}^{m} F_{\mathbf{j}} u_{\lambda}$ by weight consideration, which is identical to $\sum \mathbb{C}(q) F_{\mathbf{i}}^{\mathbf{n}} f_{i}^{m} F_{\mathbf{j}} u_{\lambda}$ by [Kas2, Corollary 3.2.2]. This proves Lemma 4.3.2.

Lemma 4.3.3 We have $U_{q}^{+}\left(\mathfrak{g}_{J}\right)\left(e_{i} U_{q}^{+}\left(\mathfrak{g}_{J}\right)\right)^{a} u_{w \lambda}=\sum_{\mathbf{n}, \mathbf{m}} \mathbb{C}(q) F_{\mathbf{i}}^{\mathbf{n}} F_{\mathbf{j}}^{\mathbf{m}} u_{\lambda}$.

Proof. If we set $m=0$ in the proof of Lemma 4.3.2, then the same argument shows that

$$
U_{q}^{+}\left(\mathfrak{g}_{J}\right)\left(e_{i} U_{q}^{+}\left(\mathfrak{g}_{J}\right)\right)^{a} u_{w \lambda}=U_{q}^{+}\left(\mathfrak{g}_{r e}\right) \sum \mathbb{C}(q) F_{\mathbf{i}}^{\mathbf{n}} F_{\mathbf{j}}^{\mathbf{m}} u_{\lambda}
$$

Now, it is easily seen that the right-hand side of (9) is identical to $\sum \mathbb{C}(q) F_{\mathbf{i}}^{\mathbf{n}} F_{\mathbf{j}}^{\mathbf{m}} u_{\lambda}$ by direct calculation. This proves Lemma 4.3.3.

\subsection{The maps $\Psi_{\lambda, w}$ and $\Phi_{\lambda, w}$}

Let $\tilde{\mathfrak{g}}_{r e} \subset \tilde{\mathfrak{g}}$ denote the Levi subalgebra corresponding to $\tilde{I}^{r e}:=\{(i, 1)\}_{i \in I^{r e}}$. Since $\mathfrak{g}_{r e}$ (see $\S 4.3)$ and $\tilde{\mathfrak{g}}_{r e}$ are ordinary Kac-Moody algebras with the same Cartan datum, there exists an embedding of $\mathbb{C}(q)$-algebras:

$$
\Psi_{r e}: U_{q}\left(\mathfrak{g}_{r e}\right) \hookrightarrow U_{q}\left(\tilde{\mathfrak{g}}_{r e}\right), e_{i} \longmapsto e_{(i, 1)}, f_{i} \longmapsto f_{(i, 1)}, q^{\alpha_{i}^{\vee} \longmapsto} q^{\tilde{\alpha}_{(i, 1)}^{\vee}}
$$

According to the minimal dominant reduced expression $w=w_{k} r_{i_{k}}^{a_{k}} \cdots w_{1} r_{i_{1}}^{a_{1}} w_{0}$, the element $\tilde{w} \in$ $\widetilde{W}\left(\right.$ see Remark 4.1.2) is expressed as $\tilde{w}=\tilde{w}_{k} R_{\left(i_{k}, \boldsymbol{a}_{k}\right)} \cdots \tilde{w}_{1} R_{\left(i_{1}, \boldsymbol{a}_{1}\right)} \tilde{w}_{0}$, with $\boldsymbol{a}_{s}=\left(a_{s}, \ldots, 2,1\right), 1 \leq$ 
$s \leq k$, where each $\tilde{w}_{s} \in \widetilde{W}_{r e}$ corresponds to $w_{s} \in \mathcal{W}_{r e}$ via the isomorphism (3) in $\S 3.2$ and we write $(i, \mathbf{m})=\left(\left(i, m_{l}\right), \ldots,\left(i, m_{1}\right)\right)$ if $\mathbf{m}=\left(m_{l}, \ldots, m_{1}\right)$; recall the notation $R_{(\mathbf{i}, \mathbf{m})}=r_{\left(i_{l}, m_{l}\right)} \cdots r_{\left(i_{1}, m_{1}\right)}$. Note that this expression for $\tilde{w}$ has properties similar to those in Lemma 4.2.2 for $\tilde{\lambda} \in \widetilde{P}^{+}$. Let $\widetilde{V}_{\tilde{w}}(\tilde{\lambda})$ denote the Demazure submodule, corresponding to $\tilde{w}$, of the irreducible highest weight $U_{q}(\tilde{\mathfrak{g}})$-module $\tilde{V}(\tilde{\lambda})$ of highest weight $\tilde{\lambda}$. We can think of $\widetilde{V}_{\tilde{w}}(\tilde{\lambda})$ as a $U_{q}^{+}\left(\mathfrak{g}_{r e}\right)$-module via $\Psi_{\text {re }}$ (see (10) ) .

In this subsection, we construct a $U_{q}^{+}\left(\mathfrak{g}_{r e}\right)$-linear maps $\Psi_{\lambda, w}: V_{w}(\lambda) \hookrightarrow \widetilde{V}_{\tilde{w}}(\tilde{\lambda})$ and $\Phi_{\lambda, w}$ : $\widetilde{V}_{\tilde{w}}(\tilde{\lambda}) \rightarrow V_{w}(\lambda)$ such that $\Phi_{\lambda, w} \circ \Psi_{\lambda, w}=\operatorname{id}_{V_{w}(\lambda)}$. For this purpose, we first study the $U_{q}^{+}\left(\mathfrak{g}_{r e}\right)$ module structure of $V_{w}(\lambda)$ and $\widetilde{V}_{\tilde{w}}(\tilde{\lambda})$. As in $\S 4.3$, define $F_{\tilde{v}}^{\mathbf{m}}, \widetilde{F}_{\tilde{v}}^{\mathbf{m}}$ and $F_{(\mathbf{i}, \mathbf{n})}^{\mathbf{m}}$ for $\tilde{v} \in \widetilde{W}$ and $(\mathbf{i}, \mathbf{n}) \in \widetilde{\mathcal{I}}$. Set $F \frac{\mathbf{m}}{\tilde{w}_{k}, \ldots, \tilde{w}_{s}}:=F_{\tilde{w}_{k}}^{\mathbf{m}_{k}} \cdots F_{\tilde{w}_{s}}^{\mathbf{m}_{s}} \in U_{q}^{-}(\tilde{\mathfrak{g}}), \underline{\mathbf{m}}=\left(\mathbf{m}_{t}\right)_{t=s}^{k} \in \prod_{t=s}^{k} \mathbb{Z}_{\geq 0}^{\ell\left(\tilde{w}_{t}\right)}$, and $\tilde{u}_{\underline{\mathbf{n}}}:=$ $F_{\left(i_{s}, \mathbf{n}_{s}\right)} F_{\tilde{w}_{s-1}} \cdots F_{\left(i_{1}, \mathbf{n}_{1}\right)} F_{\tilde{w}_{0}} \tilde{u}_{\tilde{\lambda}} \in \tilde{V}(\tilde{\lambda}), \underline{\mathbf{n}}=\left(\mathbf{n}_{t}\right)_{t=1}^{s}, \mathbf{n}_{t} \subset\left\{1,2, \ldots, a_{t}\right\}$. Here we understand that $\tilde{u}_{\underline{\mathbf{n}}}=\tilde{u}_{\tilde{\lambda}}$ if $s=0$. Note that if $\# \mathbf{n}_{t}>1$, then $a_{i_{t}, i_{t}}=0$ by Lemma 4.2.1, and hence all the $f_{\left(i_{t}, m\right)}, m \geq 1$, commute with each other. Therefore, the element $F_{\left(i_{t}, \mathbf{n}_{t}\right)}$ is independent of the choice of an ordering of the elements in $\mathbf{n}_{t}$. Also, set $F \frac{\mathbf{m}}{w_{k}, \ldots, w_{s}}:=F_{w_{k}}^{\mathbf{m}_{k}} \cdots F_{w_{s}}^{\mathbf{m}_{s}} \in U_{q}^{-}(\mathfrak{g})$ and $u_{\underline{\mathbf{n}}}:=f_{i_{s}}^{\# \mathbf{n}_{s}} F_{w_{s-1}} \cdots f_{i_{1}}^{\# \mathbf{n}_{1}} F_{w_{0}} u_{\lambda} \in V(\lambda)$; note that $u_{\underline{\mathbf{n}}}$ depends only on the cardinalities $\# \mathbf{n}_{t}$, $1 \leq t \leq s$. Hence we write $u_{\underline{\epsilon}}:=u_{\underline{\mathbf{n}}}$ for $\underline{\boldsymbol{\epsilon}}=\left(\epsilon_{t}\right)_{t=1}^{s}$ if $\epsilon_{t}=\# \mathbf{n}_{t}, 1 \leq t \leq s$. If $\mathbf{n}_{t} \neq \emptyset$ for $1 \leq t \leq s$, then $u_{\underline{\mathbf{n}}}$ (resp., $\tilde{u}_{\underline{\mathbf{n}}}$ ) is a highest weight vector for $U_{q}\left(\mathfrak{g}_{r e}\right)$ (resp., $U_{q}\left(\tilde{\mathfrak{g}}_{r e}\right)$ ) of weight $r_{i_{s}}^{\# \mathbf{n}_{s}} w_{s-1} \cdots r_{i_{1}}^{\# \mathbf{n}_{1}} w_{0}(\lambda) \in P$ (resp., $R_{\left(i_{s}, \mathbf{n}_{s}\right)} \tilde{w}_{s-1} \cdots R_{\left(i_{1}, \mathbf{n}_{1}\right)} \tilde{w}_{0}(\tilde{\lambda}) \in \widetilde{P}$ ) by Lemma 4.2.2. Moreover, in this case, we have $\alpha_{i}^{\vee}\left(r_{i_{s}}^{\# \mathbf{n}_{s}} w_{s-1} \cdots r_{i_{1}}^{\# \mathbf{n}_{1}} w_{0}(\lambda)\right)=\tilde{\alpha}_{(i, 1)}^{\vee}\left(R_{\left(i_{s}, \mathbf{n}_{s}\right)} \tilde{w}_{s-1} \cdots R_{\left(i_{1}, \mathbf{n}_{1}\right)} \tilde{w}_{0}(\tilde{\lambda})\right)$ for all $i \in I^{r e}$, and hence an isomorphism $U_{q}\left(\mathfrak{g}_{r e}\right) u_{\underline{\mathbf{n}}} \cong U_{q}\left(\tilde{\mathfrak{g}}_{r e}\right) \tilde{u}_{\underline{\mathbf{n}}}$ of $U_{q}\left(\mathfrak{g}_{r e}\right)$-modules via $\Psi_{r e}$ (see (101)) .

Lemma 4.4.1 As $U_{q}^{+}\left(\mathfrak{g}_{r e}\right)$-modules, $V_{w}(\lambda)$ and $\widetilde{V}_{\tilde{w}}(\tilde{\lambda})$ decompose as follows:

(1) $V_{w}(\lambda)=\bigoplus_{s=0}^{k} \bigoplus_{\underline{\epsilon}}\left(\sum_{\underline{\mathbf{m}}} \mathbb{C}(q) F \overline{\mathbf{m}}_{w_{k}}, \ldots, w_{s} u_{\underline{\boldsymbol{\epsilon}}}\right)$, where the summation is over all $\underline{\mathbf{m}}=\left(\mathbf{m}_{t}\right)_{t=s}^{k} \in$ $\prod_{t=s}^{k} \mathbb{Z}_{\geq 0}^{\ell\left(w_{t}\right)}$ and all $\underline{\boldsymbol{\epsilon}}=\left(\epsilon_{t}\right)_{t=1}^{s}$, with $1 \leq \epsilon_{t} \leq a_{t}, 1 \leq t \leq s$, for each $0 \leq s \leq k$.

(2) $\widetilde{V}_{\tilde{w}}(\tilde{\lambda})=\bigoplus_{s=0}^{k} \bigoplus_{\underline{\mathbf{n}}}\left(\sum_{\underline{\mathbf{m}}} \mathbb{C}(q) F_{\tilde{w}_{k}, \ldots, \tilde{w}_{s}} \tilde{u}_{\underline{\mathbf{n}}}\right)$, where the summation is over all $\underline{\mathbf{m}}=\left(\mathbf{m}_{t}\right)_{t=s}^{k} \in$ $\prod_{t=s}^{k} \mathbb{Z}_{\geq 0}^{\ell\left(\tilde{w}_{t}\right)}$ and all $\underline{\mathbf{n}}=\left(\mathbf{n}_{t}\right)_{t=1}^{s}$, with $\emptyset \neq \mathbf{n}_{t} \subset\left\{1,2, \ldots, a_{t}\right\}, 1 \leq t \leq s$, for each $0 \leq s \leq k$.

Proof. We give a proof only for (1); the proof for (2) is similar. By Lemma 4.2 .2 and Proposition 4.3 .1 (1), it is easily seen that $\left\{u_{\underline{\epsilon}}\right\}_{\underline{\epsilon}}$, where the $\underline{\boldsymbol{\epsilon}}$ runs as in Lemma 4.4.1 (1), forms a complete set of representatives of linearly independent $U_{q}\left(\mathfrak{g}_{r e}\right)$-highest weight vectors in $V_{w}(\lambda)$. Therefore, the summation $\sum_{s=0}^{k} \sum_{\underline{\boldsymbol{\epsilon}}}\left(\sum_{\underline{\mathbf{m}}} \mathbb{C}(q) F_{w_{k}}, \ldots, w_{s} u_{\underline{\boldsymbol{\epsilon}}}\right)$ is a direct sum with respect to $s$ and $\underline{\boldsymbol{\epsilon}}$. Now, the equality in (1) follows from Proposition 4.3.1 (1). Also, from the arguments in $\S 4.3$, we know that each direct summand $\sum_{\underline{\mathbf{m}}} \mathbb{C}(q) F \frac{\mathbf{m}}{w_{k}, \ldots, w_{s}} u_{\underline{\epsilon}}$ is stable under the action of $U_{q}^{+}\left(\mathfrak{g}_{r e}\right)$. Thus, the expression for $V_{w}(\lambda)$ in Lemma 4.4.1 (1) is a decomposition as $U_{q}^{+}\left(\mathfrak{g}_{r e}\right)$-modules. 
By using the descriptions of $V_{w}(\lambda)$ and $\widetilde{V}_{\tilde{w}}(\tilde{\lambda})$ in Lemma 4.4.1, define $\Phi_{\lambda, w}: \widetilde{V}_{\tilde{w}}(\tilde{\lambda}) \rightarrow V_{w}(\lambda)$ by

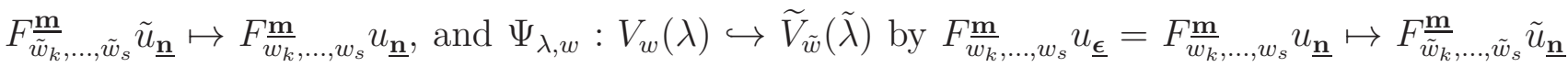
for $\underline{\mathbf{n}}=\left(\mathbf{n}_{t}\right)_{t=1}^{s}, \mathbf{n}_{t}=\left\{1,2, \ldots, \epsilon_{t}\right\}, 1 \leq \epsilon_{t} \leq a_{t}, 1 \leq t \leq s$, with $\underline{\boldsymbol{\epsilon}}=\left(\epsilon_{t}\right)_{t=1}^{s}$. Since $U_{q}\left(\tilde{\mathfrak{g}}_{r e}\right) \tilde{u}_{\underline{\mathbf{n}}} \cong$ $U_{q}\left(\mathfrak{g}_{r e}\right) u_{\underline{\mathbf{n}}}$ as $U_{q}\left(\mathfrak{g}_{r e}\right)$-modules, we deduce that $\sum_{\underline{\mathbf{m}}} \mathbb{C}(q) F{\overline{w_{k}}, \ldots, \tilde{w}_{s}}_{\tilde{u}_{\underline{\mathbf{n}}}} \cong \sum_{\underline{\mathbf{m}}} \mathbb{C}(q) F \frac{\mathbf{m}}{w_{k}, \ldots, w_{s}} u_{\underline{\mathbf{n}}}$ as $U_{q}^{+}\left(\mathfrak{g}_{r e}\right)$-modules via the map $\Phi_{\lambda, w}$ for all $\underline{\mathbf{n}}$ as in Lemma 4.4 .1 (2). Thus, $\Phi_{\lambda, w}$ is well-defined, surjective, and $U_{q}^{+}\left(\mathfrak{g}_{r e}\right)$-linear. Also, we can verify that $\Psi_{\lambda, w}$ is well-defined, injective, and $U_{q}^{+}\left(\mathfrak{g}_{r e}\right)$-linear. Note that $\Psi_{\lambda, w}$ is also surjective (and hence an isomorphism) if and only if $a_{1}=\cdots=a_{k}=1$. By the definitions, we have $\Phi_{\lambda, w} \circ \Psi_{\lambda, w}=\operatorname{id}_{V_{w}(\lambda)}$.

\subsection{Global bases and diagram automorphisms}

In this subsection, we recall some fundamental properties of the global basis of $\widetilde{V}_{\tilde{w}}(\tilde{\lambda})$. Also, we study certain symmetries of $\widetilde{V}_{\tilde{w}}(\tilde{\lambda})$, which come from diagram automorphisms of $\tilde{\mathfrak{g}}$.

Let $\widetilde{B}(\tilde{\lambda})$ and $\left\{\widetilde{G}_{\tilde{\lambda}}\left(b^{\prime}\right)\right\}_{b^{\prime} \in \widetilde{B}(\tilde{\lambda})}$ denote the crystal basis and the global basis of $\widetilde{V}(\tilde{\lambda})$ with the crystal lattice $\widetilde{L}(\tilde{\lambda})$, and let $\widetilde{V}(\tilde{\lambda})^{\mathbf{A}} \subset \tilde{V}(\tilde{\lambda})$ denote the A-form of $\tilde{V}(\tilde{\lambda})$. If we set

$$
\begin{aligned}
& \widetilde{B}_{\tilde{w}}(\tilde{\lambda}):=\left\{\widetilde{F}_{\tilde{w}_{k}}^{\mathbf{m}_{k}} \widetilde{F}_{\left(i_{k}, \mathbf{n}_{k}\right)} \cdots \widetilde{F}_{\tilde{w}_{1}}^{\mathbf{m}_{1}} \widetilde{F}_{\left(i_{1}, \mathbf{n}_{1}\right)} \widetilde{F}_{\tilde{w}_{0}}^{\mathbf{m}_{0}} \tilde{u}_{\tilde{\lambda}} \bmod q \widetilde{L}(\tilde{\lambda}) \mid\right. \\
&\left.\left(\mathbf{m}_{s}\right)_{s=0}^{k} \in \prod_{s=0}^{k} \mathbb{Z}_{\geq 0}^{\ell\left(\tilde{w}_{s}\right)}, \mathbf{n}_{t} \subset\left\{1,2, \ldots, a_{t}\right\}, 1 \leq t \leq k\right\} \backslash\{0\} \subset \widetilde{B}(\tilde{\lambda}),
\end{aligned}
$$

then we have $\widetilde{V}_{\tilde{w}}(\tilde{\lambda})=\bigoplus_{b^{\prime} \in \widetilde{B}_{\tilde{w}}(\tilde{\lambda})} \mathbb{C}(q) \widetilde{G}_{\tilde{\lambda}}\left(b^{\prime}\right)$ by $\left[\right.$ Kas2, Proposition 3.2.3]. Let $B_{w}(\lambda) \subset B(\lambda)$ denote the inverse image of $\widetilde{B}_{\tilde{w}}(\tilde{\lambda})$ under the embedding $B(\lambda) \hookrightarrow \widetilde{B}(\tilde{\lambda})$ of (2) in $\S 2.5$, and $\widetilde{B}_{\tilde{w}}^{0}(\tilde{\lambda}) \subset \widetilde{B}(\tilde{\lambda})$ the image of $B_{w}(\lambda)$ under this embedding. Then, these subsets can be written as

$$
\begin{aligned}
& B_{w}(\lambda)=\{\left\{\widetilde{F}_{w_{k}}^{\mathbf{m}_{k}} \tilde{f}_{i_{k}}^{\epsilon_{k}} \cdots \widetilde{F}_{w_{1}}^{\mathbf{m}_{1}} \tilde{f}_{i_{1}}^{\epsilon_{1}} \widetilde{F}_{w_{0}}^{\mathbf{m}_{0}} u_{\lambda} \bmod q L(\lambda) \mid\right. \\
&\left.\left(\mathbf{m}_{s}\right)_{s=0}^{k} \in \prod_{s=0}^{k} \mathbb{Z}_{\geq 0}^{\ell\left(w_{s}\right)}, 0 \leq \epsilon_{t} \leq a_{t}, 1 \leq t \leq k\right\} \backslash\{0\}, \text { and } \\
& \widetilde{B}_{\tilde{w}}^{0}(\tilde{\lambda})=\left\{\widetilde{F}_{\tilde{w}_{k}}^{\mathbf{m}_{k}} \widetilde{F}_{\left(i_{k}, \mathbf{n}_{k}\right)} \cdots \widetilde{F}_{\tilde{w}_{1}}^{\mathbf{m}_{1}} \widetilde{F}_{\left(i_{1}, \mathbf{n}_{1}\right)} \widetilde{F}_{\tilde{w}_{0}}^{\mathbf{m}_{0}} \tilde{u}_{\tilde{\lambda}} \bmod q \widetilde{L}(\tilde{\lambda}) \mid\right. \\
&\left.\left(\mathbf{m}_{s}\right)_{s=0}^{k} \in \prod_{s=0}^{k} \mathbb{Z}_{\geq 0}^{\ell\left(\tilde{w}_{s}\right)}, \mathbf{n}_{t}=\left\{1,2, \ldots, \epsilon_{t}\right\}, 0 \leq \epsilon_{t} \leq a_{t}, 1 \leq t \leq k\right\} \backslash\{0\} .
\end{aligned}
$$

In $\S 5$, we will show that the subset $B_{w}(\lambda) \subset B(\lambda)$ satisfies the condition in Theorem 3 ,

Let us set $\tilde{J}_{s}:=\left\{\left(i_{s}, m\right) \in \tilde{I} \mid 1 \leq m \leq a_{s}\right\}$ for $1 \leq s \leq k$, with each $i_{s} \in I^{i m}$ and $a_{s}$ appearing in the expression $w=w_{k} r_{i_{k}}^{a_{k}} \cdots w_{1} r_{i_{1}}^{a_{1}} w_{0}$. Also, we set $\Omega_{\lambda, w}:=\prod_{s=1}^{k} \mathfrak{S}\left(\tilde{J}_{s}\right) \subset \Omega$, where $\mathfrak{S}\left(\tilde{J}_{s}\right)$ denote the permutation group on $\tilde{J}_{s}$ (see $\S 2.2$ ). Note that the action of $\omega \in \Omega_{\lambda, w}$ on $\tilde{I}$ is expressed as: $\omega(j, n)=(j, n)$ if $(j, n) \notin \bigcup_{s=1}^{k} \tilde{J}_{s}$, and $\omega\left(i_{s}, m\right)=\left(i_{s}, m^{\prime}\right)$ for some $1 \leq m^{\prime} \leq a_{s}$ if $\left(i_{s}, m\right) \in \tilde{J}_{s}$. From this observation, we can show that $\widetilde{V}_{\tilde{w}}(\tilde{\lambda})$ and $\widetilde{B}_{\tilde{w}}(\tilde{\lambda})$ is stable under the action of $\Omega_{\lambda, w}$. By using the description of Lemma 4.4.1 for $\widetilde{V}_{\tilde{w}}(\tilde{\lambda})$, each $\omega \in \Omega_{\lambda, w}$ sends the element 
$F \frac{\mathbf{m}}{\tilde{w}_{k}, \ldots, \tilde{w}_{s}} \tilde{u}_{\underline{\mathbf{n}}}$, with $\underline{\mathbf{n}}=\left(\mathbf{n}_{s}, \ldots, \mathbf{n}_{1}\right)$, to the element of the form $F \frac{\mathbf{m}}{\tilde{w}_{k}, \ldots, \tilde{w}_{s}} \tilde{u}_{\underline{\mathbf{n}}^{\prime}}$, with $\underline{\mathbf{n}^{\prime}}=\left(\mathbf{n}_{s}^{\prime}, \ldots, \mathbf{n}_{1}^{\prime}\right)$ such that $\# \mathbf{n}_{t}=\# \mathbf{n}_{t}^{\prime}$ for $1 \leq t \leq s$. Note that this expression for the action of $\Omega_{\lambda, w}$ on $\widetilde{V}_{\tilde{w}}(\tilde{\lambda})$ shows that $\Phi_{\lambda, w} \circ \omega=\Phi_{\lambda, w}$ for all $\omega \in \Omega_{\lambda, w}$. Also, for each $\underline{\mathbf{n}}^{\prime}=\left(\mathbf{n}_{s}^{\prime}, \ldots, \mathbf{n}_{1}^{\prime}\right)$ with $\# \mathbf{n}_{t}^{\prime}=\# \mathbf{n}_{t}$ for $1 \leq t \leq s$, we can find $\omega \in \Omega_{\lambda, w}$ such that $\omega\left(F \frac{\mathbf{m}}{\underline{w_{k}}, \ldots, \tilde{w}_{s}} \tilde{u}_{\underline{\mathbf{n}}}\right)=F \frac{\mathbf{m}}{\tilde{w}_{k}, \ldots, \tilde{w}_{s}} \tilde{u}_{\underline{\mathbf{n}}^{\prime}}$. In particular, if we take $\underline{\mathbf{n}^{\prime}}=\left(\mathbf{n}_{s}^{\prime}, \ldots, \mathbf{n}_{1}^{\prime}\right)$ such that $\mathbf{n}_{t}^{\prime}=\left\{1,2, \ldots, \# \mathbf{n}_{t}\right\}$ for $1 \leq t \leq s$, then the corresponding $\omega \in \Omega_{\lambda, w}$ (not necessarily unique) sends $F \widetilde{w}_{\tilde{w}_{k}, \ldots, \tilde{w}_{s}} \tilde{u}_{\underline{\mathbf{n}}}$ into the image of the map $\Psi_{\lambda, w}$. By the same argument, for each $b^{\prime} \in \widetilde{B}_{\tilde{w}}(\tilde{\lambda})$, we can find $\omega \in \Omega_{\lambda, w}$ such that $\omega\left(b^{\prime}\right)$ lies in $\widetilde{B}_{\tilde{w}}^{0}(\tilde{\lambda})$. Namely, we have $\widetilde{B}_{\tilde{w}}(\tilde{\lambda})=\Omega_{\lambda, w} \widetilde{B}_{\tilde{w}}^{0}(\tilde{\lambda})$. Note that $\widetilde{G}_{\tilde{\lambda}} \circ \omega=\omega \circ \widetilde{G}_{\tilde{\lambda}}$ for all $\omega \in \Omega_{\lambda, w}$ (see $[\mathbf{S}$, Lemma 3.4]).

\subsection{Compatibility with the global bases}

This subsection is devoted to the proof of Proposition 4.6.1 below. For $b^{\prime} \in \widetilde{B}_{\tilde{w}}(\tilde{\lambda})$, we set $\mathbb{G}\left(b^{\prime}\right):=\Phi_{\lambda, w}\left(\widetilde{G}_{\tilde{\lambda}}\left(b^{\prime}\right)\right) \in V_{w}(\lambda)$.

Proposition 4.6.1 The element $\mathbb{G}\left(b^{\prime}\right) \in V_{w}(\lambda), b^{\prime} \in \widetilde{B}_{\tilde{w}}(\tilde{\lambda})$, is a global basis element.

Proof. To complete the proof, in view of the characterization of a global basis element (see §2.1), we must show (i) $\overline{\mathbb{G}\left(b^{\prime}\right)}=\mathbb{G}\left(b^{\prime}\right)$, (ii) $\mathbb{G}\left(b^{\prime}\right) \in V(\lambda)^{\mathbf{A}} \cap L(\lambda)$, and (iii) $\mathbb{G}\left(b^{\prime}\right)$ mod $q L(\lambda) \in B(\lambda) \backslash$

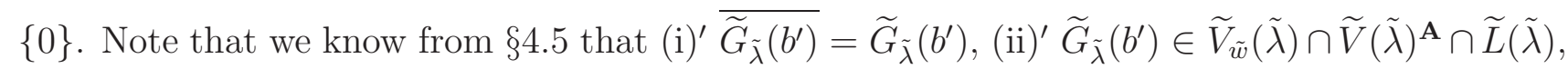
and (iii) $\widetilde{G}_{\tilde{\lambda}}\left(b^{\prime}\right) \equiv b^{\prime} \bmod q \widetilde{L}(\tilde{\lambda})$. Then, the equality (i) follows from (i)'. Obviously, we have $\Phi_{\lambda, w}\left(\widetilde{V}_{\tilde{w}}(\tilde{\lambda}) \cap \tilde{V}(\tilde{\lambda})^{\mathbf{A}}\right) \subset V(\lambda)^{\mathbf{A}}$. Therefore, by (ii)', it suffices to verify that $\Phi_{\lambda, w}\left(\widetilde{V}_{\tilde{w}}(\tilde{\lambda}) \cap \widetilde{L}(\tilde{\lambda})\right) \subset$ $L(\lambda)$ to show (ii). For this purpose, the following is enough:

Claim 1 If $\widetilde{F}_{(\mathbf{i}, \mathbf{m})}$ is of the form $\widetilde{F}_{\tilde{w}_{k}}^{\mathbf{m}_{k}} \widetilde{F}_{\left(i_{k}, \mathbf{n}_{k}\right)} \cdots \widetilde{F}_{\tilde{w}_{1}}^{\mathbf{m}_{1}} \widetilde{F}_{\left(i_{k}, \mathbf{n}_{1}\right)} \widetilde{F}_{\tilde{w}_{0}}^{\mathbf{m}_{0}}$, with $\left(\mathbf{m}_{s}\right)_{s=0}^{k} \in \prod_{s=0}^{k} \mathbb{Z}_{\geq 0}^{\ell\left(\tilde{w}_{s}\right)}, \mathbf{n}_{t} \subset$ $\left\{1,2, \ldots, a_{t}\right\}, 1 \leq t \leq k$, then we have $\Phi_{\lambda, w}\left(\widetilde{F}_{(\mathbf{i}, \mathbf{m})} \tilde{u}_{\tilde{\lambda}}\right)=\widetilde{F}_{\mathbf{i}} u_{\lambda}$.

Proof of Claim 1. Set $v=\widetilde{F}_{(\mathbf{i}, \mathbf{m})} \tilde{u}_{\tilde{\lambda}}$. We proceed by induction on the length $l$ of $(\mathbf{i}, \mathbf{m})=$ $\left(\left(i_{s}, m_{s}\right)\right)_{s=1}^{l}$. We assume that $\Phi_{\lambda, w}(v)=\widetilde{F}_{\mathbf{i}} u_{\lambda}$, and show that $\Phi_{\lambda, w}\left(\tilde{f}_{(i, m)} v\right)=\tilde{f}_{i} \widetilde{F}_{\mathbf{i}} u_{\lambda}$ for all $(i, m) \in \tilde{I}$ such that $\tilde{f}_{(i, m)} \widetilde{F}_{(\mathbf{i}, \mathbf{m})}$ is also of the form given as in Claim 1 .

Case 1: $i \in I^{i m}$.

Since $(i, m)$ does not appear in $(\mathbf{i}, \mathbf{m})$, we have $v \in \operatorname{Ker}\left(e_{(i, m)}\right)$ and hence $\tilde{f}_{(i, m)} v=f_{(i, m)} v$. Hence we deduce that $\Phi_{\lambda, w}\left(\tilde{f}_{(i, m)} v\right)=\Phi_{\lambda, w}\left(f_{(i, m)} v\right)=f_{i} \Phi_{\lambda, w}(v)$. Also, we have $\tilde{f}_{i} \Phi_{\lambda, w}(v)=f_{i} \Phi_{\lambda, w}(v)$ since $\tilde{f}_{i}=f_{i}$ if $i \in I^{i m}$ (see $\S 2.1$ ). Thus the assertion follows in this case.

Case 2: $i \in I^{r e}$.

Let $v=\sum_{n \geq 0} f_{(i, 1)}^{(n)} v_{n}$, with $v_{n} \in \operatorname{Ker}\left(e_{(i, 1)}\right)$, be the $(i, 1)$-string decomposition (see $\left.\S 2.1\right)$. Since $\widetilde{V}_{\tilde{w}}(\tilde{\lambda})$ is stable under the action of $e_{(i, 1)}$, we can show, by induction on $n$, that $v_{n}, f_{(i, 1)}^{(n)} v_{n} \in \widetilde{V}_{\tilde{w}}(\tilde{\lambda})$ 
for all $n \geq 0$. Hence the equality $\Phi_{\lambda, w}(v)=\sum_{n \geq 0} f_{i}^{(n)} \Phi_{\lambda, w}\left(v_{n}\right)$ holds. Also, we have $\Phi_{\lambda, w}\left(v_{n}\right) \in$ $\operatorname{Ker}\left(e_{i}\right)$ since $e_{i} \Phi_{\lambda, w}\left(v_{n}\right)=\Phi_{\lambda, w}\left(e_{(i, 1)} v_{n}\right)=0$. Therefore, this expression for $\Phi_{\lambda, w}(v)$ gives the $i$-string decomposition. Thus we have $\tilde{f}_{i} \Phi_{\lambda, w}(v)=\sum_{n \geq 0} f_{i}^{(n+1)} \Phi_{\lambda, w}\left(v_{n}\right)$, which is also identical to the image of the element $\tilde{f}_{(i, 1)} v=\sum_{n \geq 0} f_{(i, 1)}^{(n+1)} v_{n}$ under the map $\Phi_{\lambda, w}$. This proves Claim 11.

Now, let us show (iii). Note that Claim 1 above shows that $\Phi_{\lambda, w}$ induces the map $\bar{\Phi}_{\lambda, w}$ : $\widetilde{B}_{\tilde{w}}(\tilde{\lambda}) \rightarrow B(\lambda)$. Moreover, it is easily seen that the image of this map is $B_{w}(\lambda)$, and that the equality $\bar{\Phi}_{\lambda, w} \circ^{\sim}=\operatorname{id}_{B_{w}(\lambda)}$ holds, where $\sim$ denotes (the restriction of) the map of (2) in $\S 2.5$. From these observations, we see that if we take an element $\tilde{b} \in \widetilde{B}_{\tilde{w}}^{0}(\tilde{\lambda})$, which is the image of an element $b \in B_{w}(\lambda)$ under the map $\sim$, then we have $\mathbb{G}(\tilde{b})=\Phi_{\lambda, w}\left(\widetilde{G}_{\tilde{\lambda}}(\tilde{b})\right) \equiv \bar{\Phi}_{\lambda, w}(\tilde{b}) \equiv b$ $\bmod q L(\lambda)$, where the second equality is due to (iii)'. For a general element $b^{\prime} \in \widetilde{B}_{\tilde{w}}(\tilde{\lambda})$, there exists a diagram automorphism $\omega \in \Omega_{\lambda, w}$ such that $\omega^{-1}\left(b^{\prime}\right)=\tilde{b} \in \widetilde{B}_{\tilde{w}}^{0}(\tilde{\lambda})$, where $\tilde{b}$ is as above. Since $\widetilde{G}_{\tilde{\lambda}} \circ \omega=\omega \circ \widetilde{G}_{\tilde{\lambda}}$ and $\Phi_{\lambda, w} \circ \omega=\Phi_{\lambda, w}$ (see $\left.\S 4.5\right)$, we see that $\mathbb{G}\left(b^{\prime}\right)=\Phi_{\lambda, w}\left(\widetilde{G}_{\tilde{\lambda}}(\omega(\tilde{b}))\right)=$ $\Phi_{\lambda, w}\left(\omega\left(\widetilde{G}_{\tilde{\lambda}}(\tilde{b})\right)\right)=\Phi_{\lambda, w}\left(\widetilde{G}_{\tilde{\lambda}}(\tilde{b})\right)=\mathbb{G}(\tilde{b})$. Therefore, we deduce that $\mathbb{G}\left(b^{\prime}\right) \equiv b \bmod q L(\lambda)$. This completes the proof of Proposition 4.6.1.

In the same way as above, we can also show that the map $\Psi_{\lambda, w}$ has properties similar to those for $\Phi_{\lambda, w}$, and hence induces the map $\bar{\Psi}_{\lambda, w}: B_{w}(\lambda) \rightarrow \widetilde{B}_{\tilde{w}}(\tilde{\lambda})$. In fact, this is identical to the map .

Corollary 4.6.2 With the notation above, the following hold.

(1) The maps $\Phi_{\lambda, w}$ and $\Psi_{\lambda, w}$ induce maps $\bar{\Phi}_{\lambda, w}: \widetilde{B}_{\tilde{w}}(\tilde{\lambda}) \rightarrow B_{w}(\lambda)$ and $\bar{\Psi}_{\lambda, w}: B_{w}(\lambda) \hookrightarrow \widetilde{B}_{\tilde{w}}(\tilde{\lambda})$ such that $\bar{\Phi}_{\lambda, w} \circ \bar{\Psi}_{\lambda, w}=\mathrm{id}_{B_{w}(\lambda)}$.

(2) The equalities $G_{\lambda} \circ \bar{\Phi}_{\lambda, w}=\Phi_{\lambda, w} \circ \widetilde{G}_{\tilde{\lambda}}$ and $\widetilde{G}_{\tilde{\lambda}} \circ \bar{\Psi}_{\lambda, w}=\Psi_{\lambda, w} \circ G_{\lambda}$ hold.

(3) Let $b \in B_{w}(\lambda), \tilde{b}=\bar{\Psi}_{\lambda, w}(b)$, and $\omega \in \Omega_{\lambda, w}$. Then, the equality $\mathbb{G}(\omega(\tilde{b}))=G_{\lambda}(b)$ holds.

\section{Proofs of main results}

\subsection{Proof of Theorem 3}

Proof of Theorem 3. If we write the element $\Psi_{\lambda, w}(v) \in \widetilde{V}_{\tilde{w}}(\tilde{\lambda})$ for $v \in V_{w}(\lambda)$ as $\Psi_{\lambda, w}(v)=$ $\sum c_{\omega(\tilde{b})} \widetilde{G}_{\tilde{\lambda}}(\omega(\tilde{b}))$, with $c_{\omega(\tilde{b})} \in \mathbb{C}(q)$, then we have $v=\Phi_{\lambda, w}\left(\Psi_{\lambda, w}(v)\right)=\sum c_{\omega(\tilde{b})} G_{\lambda}(b)$ by Corollary 4.6 .2 (3). Therefore, we conclude that $V_{w}(\lambda)=\bigoplus_{b \in B_{w}(\lambda)} \mathbb{C}(q) G_{\lambda}(b)$. This proves Theorem 3 , 


\subsection{Proof of Theorem 4}

Before starting the proof of Theorem 4 , we fix some notation. Let $\mathbb{B}_{w}(\lambda), \widetilde{\mathbb{B}}_{\tilde{w}}(\tilde{\lambda})$, and $\widetilde{\mathbb{B}}_{\tilde{w}}^{0}(\tilde{\lambda})$ denote the subsets of path crystals corresponding to $B_{w}(\lambda), \widetilde{B}_{\tilde{w}}(\tilde{\lambda})$, and $\widetilde{B}_{\tilde{w}}^{0}(\tilde{\lambda})$ via the isomorphisms $\mathbb{B}(\lambda) \cong B(\lambda)$ and $\widetilde{\mathbb{B}}(\tilde{\lambda}) \cong \widetilde{B}(\tilde{\lambda})$ of crystals, respectively. Define subsets $\mathbb{B}_{m}$ of $\mathbb{B}_{w}(\lambda)$ for $m=1,2$, by

$$
\begin{gathered}
\mathbb{B}_{1}:=\left\{F_{w_{k-1}}^{\mathbf{m}_{k-1}} f_{i_{k-1}}^{\epsilon_{k-1}} \cdots F_{w_{1}}^{\mathbf{m}_{1}} f_{i_{1}}^{\epsilon_{1}} F_{w_{0}}^{\mathbf{m}_{0}} \pi_{\lambda} \in \mathbb{B}_{w}(\lambda) \mid \mathbf{m}_{s} \in \mathbb{Z}_{\geq 0}^{\ell\left(w_{s}\right)}, 0 \leq \epsilon_{s} \leq a_{s} \text { for each } s\right\} \\
\mathbb{B}_{2}:=\left\{f_{i_{k}}^{\epsilon} \pi \in \mathbb{B}_{w}(\lambda) \mid \pi \in \mathbb{B}_{1}, 0 \leq \epsilon \leq a_{k}\right\}
\end{gathered}
$$

and set $\widetilde{\mathbb{B}}_{m}:=\bar{\Psi}_{\lambda, w}\left(\mathbb{B}_{m}\right) \subset \widetilde{\mathbb{B}}_{\tilde{w}}^{0}(\tilde{\lambda}), m=1,2$.

Proof of Theorem 4. If $w \in \mathcal{W}_{r e}$, then $\operatorname{ch} \mathbb{B}_{w}(\lambda)=\mathcal{D}_{w}\left(e^{\lambda}\right)$ by [Kas2, Proposition 3.3.5], and the assertion of Theorem 4 follows in this case. Let $w=w_{k} r_{i_{k}}^{a_{k}} \cdots w_{1} r_{i_{1}}^{a_{1}} w_{0}$ be a minimal dominant reduced expression. We assume that the equality $\operatorname{ch} \mathbb{B}_{1}=\mathcal{D}_{w_{k-1}} \mathcal{D}_{i_{k-1}}^{\left(a_{k-1}\right)} \cdots \mathcal{D}_{w_{1}} \mathcal{D}_{i_{1}}^{\left(a_{1}\right)} \mathcal{D}_{w_{0}}\left(e^{\lambda}\right)$ holds, and show that $\operatorname{ch} \mathbb{B}_{w}(\lambda)=\mathcal{D}_{w_{k}} \mathcal{D}_{i_{k}}^{\left(a_{k}\right)}\left(\operatorname{ch} \mathbb{B}_{1}\right)$.

First, we show that $\operatorname{ch} \mathbb{B}_{2}=\mathcal{D}_{i_{k}}^{\left(a_{k}\right)}\left(\operatorname{ch} \mathbb{B}_{1}\right)$. Note that $f_{i_{k}}^{\epsilon} \pi \in \mathbb{B}_{2}$ is not $\mathbf{0}$ for $\epsilon \geq 1$ if and only if $\alpha_{i_{k}}^{\vee}(\pi(1))>0$ (see [JL, Lemma 4.1.6 (1)]). Therefore, we deduce that

$$
\operatorname{ch} \mathbb{B}_{2}=\sum_{\substack{\pi \in \mathbb{B}_{1} \\ \alpha_{i_{k}}^{\vee}(\pi(1))>0}} \sum_{\epsilon=0}^{a_{k}} e^{f_{i_{k}}^{\epsilon} \pi(1)}+\sum_{\substack{\pi \in \mathbb{B}_{1} \\ \alpha_{i_{k}}(\pi(1))=0}} e^{\pi(1)},
$$

and that the right-hand side of (11) is identical to $\mathcal{D}_{i_{k}}^{\left(a_{k}\right)}\left(\operatorname{ch} \mathbb{B}_{1}\right)$ by the definition of $\mathcal{D}_{i_{k}}^{\left(a_{k}\right)}$.

Now, we show that $\operatorname{ch} \mathbb{B}_{w}(\lambda)=\mathcal{D}_{w_{k}}\left(\operatorname{ch} \mathbb{B}_{2}\right)$. By $\left[\right.$ Kas2, Proposition 3.3.5], we have $\operatorname{ch} \widetilde{\mathbb{B}}_{\tilde{w}}(\tilde{\lambda})=$ $\mathcal{D}_{\tilde{w}}\left(e^{\tilde{\lambda}}\right)=\mathcal{D}_{\tilde{w}_{k}} \mathcal{D}_{\tilde{w}_{k}^{-1}}\left(e^{\tilde{\lambda}}\right)$. Then, we can deduce that ch $\widetilde{\mathbb{B}}_{\tilde{w}}^{0}(\tilde{\lambda})=\mathcal{D}_{\tilde{w}_{k}}\left(\operatorname{ch} \widetilde{\mathbb{B}}_{2}\right)$ since $\widetilde{\mathbb{B}}_{\tilde{w}}^{0}(\tilde{\lambda})=$ $\left\{F_{\tilde{w}_{k}}^{\mathbf{m}} \eta \mid \eta \in \widetilde{\mathbb{B}}_{2}, \mathbf{m} \in \mathbb{Z}_{\geq 0}^{\ell\left(\tilde{w}_{k}\right)}\right\} \subset \widetilde{\mathbb{B}}_{\tilde{w}}(\tilde{\lambda})$, and $\widetilde{\mathbb{B}}_{2}$ has the string property (see [Jo, Lemma in $\left.\S 4.4\right]$ ). Therefore, we deduce that $\operatorname{ch} \mathbb{B}_{w}(\lambda)=\operatorname{ch} \bar{\Phi}_{\lambda, w}\left(\widetilde{\mathbb{B}}_{\tilde{w}}^{0}(\tilde{\lambda})\right)=\mathcal{D}_{w_{k}}\left(\operatorname{ch} \bar{\Phi}_{\lambda, w}\left(\widetilde{\mathbb{B}}_{2}\right)\right)=\mathcal{D}_{w_{k}}\left(\operatorname{ch} \mathbb{B}_{2}\right)$.

Consequently, we obtain $\operatorname{ch} \mathbb{B}_{w}(\lambda)=\mathcal{D}_{w_{k}} \mathcal{D}_{i_{k}}^{\left(a_{k}\right)}\left(\operatorname{ch} \mathbb{B}_{1}\right)$. This proves Theorem 4 by induction on $k$.

\section{References}

[BB] A. Björner and F. Brenti, Combinatorics of Coxeter Groups, Grad. Texts in Math., vol. 231, Springer, New York, 2005.

[Bo] R. E. Borcherds, Generalized Kac-Moody algebras, J. Algebra 115 (1988), 501-512.

[HK] J. Hong and S.-J. Kang, Introduction to Quantum Groups and Crystal Bases, Grad. Studies in Math. vol. 42, Amer. Math. Soc., Providence, RI, 2002. 
[Hu] J. E. Humphreys, Reflection Groups and Coxeter Groups, Camb. Stud. in Adv. Math., 29, Cambridge Univ. Press, Cambridge, 1990.

[I] M. Ishii, Path model for representations of generalized Kac-Moody algebras, J. Algebra 379 (2013), 277-300.

[JKK] K. Jeong, S.-J. Kang, and M. Kashiwara, Crystal bases for quantum generalized Kac-Moody algebras, Proc. Lond. Math. Soc. 90 (2005), 395-438.

[JKKS] K. Jeong, S.-J. Kang, M. Kashiwara, and D.-U. Shin, Abstract crystals for quantum generalized Kac-Moody algebras, Int. Math. Res. Not., 2007 Art. ID rnm001, 19 pages.

[Jo] A. Joseph, A decomposition theorem for Demazure crystals, J. Algebra 265 (2003) 562-578.

[JL] A. Joseph and P. Lamprou, A Littelmann path model for crystals of generalized Kac-Moody algebras, Adv. Math. 221 (2009), 2019-2058.

[Kac] V. G. Kac, Infinite Dimensional Lie Algebras, 3rd ed., Cambridge Univ. Press, Cambridge, 1990.

[Kan] S.-J. Kang, Quantum deformations of generalized Kac-Moody algebras and their modules, J. Algebra 175 (1995), 1041-1066.

[Kas1] M. Kashiwara, On crystal bases of the $q$-analogue of universal enveloping algebras, Duke Math. J. 63 (1991), 465-509.

[Kas2] M. Kashiwara, The crystal base and Littelmann's refined Demazure character formula, Duke Math. J. 71 (1993), 839-858.

[Kum] S. Kumar, Demazure character formula in arbitrary Kac-Moody setting, Invent. Math. 89 (1987), no. 2, 395-423.

[Li1] P. Littelmann, A Littlewood-Richardson rule for symmetrizable Kac-Moody algebras, Invent. Math. 116 (1994), 329-346.

[Li2] P. Littelmann, Paths and root operators in representation theory, Ann. of Math. 124 (1995), 499-525.

[Li3] P. Littelmann, Crystal graphs and Young tableaux, J. Algebra 175 (1995), no. 1, 65-87.

[M] O. Mathieu, Formules de Demazure-Weyl, et généralisation du théorème de Borel-Weil-Bott, C. R. Acad. Sci. Paris Sér. I Math. 303 (1986), no. 9, 391-394.

[NS] S. Naito and D. Sagaki, Lakshmibai-Seshadri paths fixed by a diagram automorphism, J. Algebra 245 (2001), 395-412.

[S] D. Sagaki, Crystal bases, path models, and a twining character formula for Demazure modules, Publ. Res. Inst. Math. Sci. 38 (2002), 245-264. 